\title{
A Hybrid Optimization Scheme For Self-Potential Measurements Due to Multiple Sheet-like Bodies in Arbitrary 2D Resistivity Distributions
}

Iraklis Giannakis $^{1}$ | Panagiotis Tsourlos ${ }^{2}$ | Costas Papazachos $^{2}$ | George Vargemezis ${ }^{2}$ | Antonios Giannopoulos $^{3}$ | Nikos Papadopoulos ${ }^{4}$ | Fabio Tosti ${ }^{1}$ Amir Alani ${ }^{1}$ 
Self-potential (SP) is a passive geophysical method that can be applied in a straightforward manner with minimum requirements in the field. Nonetheless, interpretation of SP data is particularly challenging due to the inherited nonuniqueness present in all potential methods. Incorporating information regarding the target of interest can facilitate interpretation and increase the reliability of the final output. In the current paper, a novel method for detecting multiple sheet-like targets is presented. A numerical framework is initially described that simulates sheet-like bodies in an arbitrary 2D resistivity distribution. A scattered field formulation based on finite-differences is employed that allows the edges of the sheet to be independent of the grid geometry. A novel analytical solution for two-layered models is derived and subsequently used to validate the accuracy of the proposed numerical scheme. Lastly, a hybrid optimization is proposed that couples linear least-squares with particle-swarm optimization (PSO) in order to effectively locate the edges of multiple sheet-like bodies. Through numerical and real data, it is proven that the hybrid optimization overcomes local minimal that occur in complex resistivity distributions and converges substantially faster compared to traditional PSO.

\section{KEYWORDS}

Inversion, Modelling, Numerical study, Passive method, Potential field
${ }^{1}$ School of Computing and Engineering, University of West London, London, St Mary's Road, W5 5RF, United Kingdom

${ }^{2}$ Department of Geophysics, School of Geology, Aristotle University of Thessaloniki, 54124, Thessaloniki, Greece

${ }^{3}$ School of Engineering, Institute for Infrastructure and Environment, The University of Edinburgh, Edinburgh, EH9 3FG, United Kingdom

${ }^{4}$ Foundation for Research and Technology, Hellas (FORTH), Institute for Mediterranean Studies, Laboratory of Geophysical-Satellite Remote Sensing and Archaeo-Environment, Melissinou and Nik Foka 130, 74100, Rethymno, Crete, Greece

\section{Correspondence}

Iraklis Giannakis, School of Computing and Engineering, University of West London, London, St Mary's Road, W5 5RF, United Kingdom

Email: iraklis.giannakis@uwl.ac.uk

Funding information 


\section{1 | INTRODUCTION}

Self-potential (SP) is a passive near-surface geophysical technique that gained a renewed popularity due to its practicality and minimum requirements (Nyquist and Corry, 2002; Minsley, 2007). SP is often used to assist electrical resistivity tomography (ERT) (Mao, 2015) and provide further information regarding the coupled flow mechanisms responsible for spontaneous voltage sources (Marshall et. al, 1959; Sato and Mooney, 1960). Numerous case studies have been reported in the literature in which SP has been successfully applied in hydrogeophysics (Birch, 1998; Naudet et. al , 2003; Jouniaux et. al, , 2009), geothermal exploration (Corwin and Hoover , 1979), volcanology (Fournier , 1989; Aizawa , 2004), coal fire detection (Shao et. al , 2014, 2017), hydrothermal ore deposits (Kawada and Kasaya, 2017) and marine mineral deposits (Heinson et. al, 2005).

Interpretation of SP is particularly challenging due to the embedded non-uniqueness present in all potential-field methods (Pedersen , 1975; Karaoulis et. al , 2014). Traditional inversion coupled with regularizers can tackle nonuniqueness (Tikhonov and Arsenin , 1977) by constraining the solution and thus reducing the optimization space. The most common regularization method in geophysics is the Occam inversion that constrains the solution to be spatially smooth (Constable et. al , 1987). In potential methods, sparse solutions are often more preferable since geological targets such as faults and ore deposits are compact discreet volumes. Last and Kubik (1983) and Portniaguine and Zhdanov (1999) suggested a regularization scheme that prefers sparse distributions with a minimum volume span. Regardless of the chosen constrain, the sensitivity of the measurements to the parameters of the model is greatly reduced with depth. Due to that, the inversion becomes biased to near-surface disturbances. This can be overcomed with a scaled sensitivity initially suggested by Li and Oldenburg $(1996,1998)$ and applied to gravity and magnetic data. The scaled sensitivity, combined with a compactness regulizer, has been successfully applied by Minsley (2007) and Minsley et. al (2007) to 3D SP data. Minsley (2007) also shown that the resistivity distribution can vastly affect the resulting source distribution and should be coherently implemented in the inversion scheme. Recently, 4D inversion schemes, combined with additional regularizers have been applied to gravity data and numerical evidences shown promising results in time-varying non-static models (Karaoulis et. al , 2014).

The scaling factor of the selected regulizer is chosen such as to balance between respecting the constrains while minimizing the missfit between the actual and the synthetic data. The L-curve method (Hansen , 1992, 1993) is an attractive choice for selecting the scaling factor in ill-posed problems and has been applied for both SP and gravity data (Minsley et. al , 2007; Karaoulis et. al , 2014). When proper scaling factors are chosen and the constrains are accurately selected, SP inversion can reliably reconstruct the source distribution in an efficient manner. Nonetheless, background noise, over or under regularizations and improper initial-guess for subsurface resistivity distribution (for SP method) can give rise to large errors due to the unstable nature of the source inversion. A more straightforward way to constrain the solution is to make direct assumptions regarding the geometry of the target. This approach tackles non-uniqueness in a direct manner and provides accurate results if the target follows the pre-assumed geometry. Pedersen (1975) suggested a method that assumes a two layered-medium with an arbitrarily interface between the two phases and applied it to gravity data. The model consists of discreet prisms and their size and densities are evaluated during the inversion. Biswas (2015) assumed that the targets are cylinders or spheres and applied global optimizers to interpret gravity data. Similar geometrical constrains assuming spherical and cylindrical sources were applied to SP method (Bhattacharya and Roy, 1981) using analytical approaches and nomograms. A special case is that of the electroencephalography (EEG) in which SP signals are processed in order to define the current distribution inside the brain. Both parametric and non-parametric approaches have been reported in EEG (Grech et. al , 2008) and single and multiple infinitesimal dipoles (up to ten) have been widely used as geometrical constrains (Grech et. al , 2008) .

A wide range of geological targets such as geothermal fields associated with faults, faults filled with water or mineral 
deposits (graphite and sulphide lodes, etc) generate self-potential anomalies that can be simulated with a pair of 2D line sources with equal strength and opposite sign (Jagannadha et. al , 1993). This formulation is often refereed to as "sheet-like body" and its suitable for simulating contacts between two different surfaces (Sharma, 1997). Sheet-like bodies have been used since the early development of SP (Meiser , 1962) and they kept their popularity throughout the years (Rao and Babu , 1983; Eppelbaum and Khesin , 2012; Essa and Elhussein , 2017) due to their simplicity and efficiency on simulating geological targets of interests (such as fault-related mineral deposits). Meiser (1962) suggested a curve matching technique assuming sheet-like bodies subject to a homogeneous half-space. Atchuta (1982) suggested an analytical approach applied in the wavenumber domain assuming sheet-like bodies in a homogeneous half-space. Rao and Babu (1983) proposed a quantitative way of interpreting SP anomalies by using limited measurement points. This approach provides fast results, nonetheless is very sensitive to noise and assumes a homogeneous half-space. Jagannadha et. al (1993) used a non-linear damped least square inversion to determine the optimized sheet-like body for a homogeneous half-space. Their approach also allows for automatic adjustment that tackles the improper manual correction of the regional field. Akgun (2001) used the Hilbert transform in order to determine the characteristics of a sheet-like body in a homogeneous and flat earth. Hesham et. al (2009) used a shallow neural network in order to establish the underlying relationship between the given SP anomalies and the characteristics of the sheet-like body. The neural architecture was trained for homogeneous half-space. Recently, global optimizers have been employed in an effort to overcome local minimal and avoid initialization during the inversion process (Biswas and Sharma, 2014; Biswas and Shashi , 2014b; Maio et. al , 2016; Dwa and Warnana, 2018).

It should be emphasized that the methods described above can be applied only to homogeneous half-spaces. Minsley (2007) supported the premise that the resistivity distribution can highly affect the interpretation of SP data and should be accurately incorporated in any detection scheme. Giannakis et. al (2012) suggested a numerical scheme that can simulate sheet-like bodies in an arbitrary 2D resistivity distribution. This technique is based on finite-differences (FD) (Dey and Morrison, 1979) with a modification that allows the sources to be grid-independent (Spitzer et. al , 1999). Grid-independence allows the edges of the sheet-like body to freely move in the spatial domain which is crucial for any optimization scheme meant to be coupled with the aforementioned numerical framework. The fact that the source can be placed in a continuous 2D space allows for an accurate estimation of the coordinates of the dipole without using two-step procedures that employ a dense sub-grid inside a main sparse one (Haas et. al. , 2013). Subsequently, a dumbed non-linear least squares method (Marquadt , 1963) is used in order to estimate the properties of the sheet-like body subject to a $2 \mathrm{D}$ resistivity distribution.

The method suggested by Giannakis et. al (2012) is constrained to a single sheet-like body and requires good initialization in order to avoid local minimal when complex resistivity distributions are used. In addition, due to the lack of analytical solutions, the reliability of the numerical scheme is not properly validated especially when applied to marine applications in which high resistivity contrasts are present. In the present paper, an analytical solution for sheet-like bodies in two-layered media is derived and furthermore used to validate the grid-independent numerical scheme. Subsequently, in order to avoid local-minimal, a particle-swarm optimization (PSO) (Kennedy and Eberhart, 1995 ) is used to estimate the coordinates of the targets. The PSO is combined with a linear inversion for estimating the intensity of the sources. This is not to be confused with hybrid schemes that use convex and global optimization in a sequential manner in which the convergence point of the global search is used as an initial point for the subsequent convex optimization (Soupios et. al , 2011; Basokur , 2007, 2011). The present technique uses least squares to describe the intensity of the sources with respect to the coordinates of the sheet-like bodies in order to reduce the optimization space. A similar hybrid PSO-linear scheme have been successfully applied for fitting multi-Debye expansions to HavriliakNegami for electromagnetic applications (Kelley et. al , 2007). The hybrid linear-PSO scheme is superior to PSO since it reduces the optimization space resulting to faster convergence and increased stability. Neither initialization of 
the model nor tuning the regularization parameters are required. Lastly, the hybrid optimization scheme, due to its increased stability, makes it possible to accurately and efficiently detect multiple sheet-like bodies for any arbitrarily $2 \mathrm{D}$ resistivity distribution. The validity and the performance of the hybrid optimization scheme are evaluated using both real (Vargemezis et. al , 2012) and numerical data from a variety of case studies including, surface measurements, borehole surveys and marine geophysics.

\section{2 | FORWARD MODEL}

The electric potential due to a 3D point source is described by Poisson's equation (Dey and Morrison , 1979)

$$
-\nabla \cdot(\sigma(x, y, z) \nabla \phi(x, y, z))=\frac{\partial \rho}{\partial t} \delta\left(x_{s}\right) \delta\left(y_{s}\right) \delta\left(z_{s}\right)
$$

where $\sigma$ is the conductivity, $\phi$ is the electric potential, $\rho$ is the charge density, $\delta$ is the delta function and $\left(x_{s}, y_{s}, z_{s}\right)$ are the coordinates of the point source. Dey and Morrison (1979) derived a 2.5D formulation that can excite a 2D resistivity distribution $\left(\frac{\partial \sigma}{\partial y}=0\right)$ with a $3 \mathrm{D}$ point source. To do so, the potential is transformed from the space domain $(x, y, z)$ to the space-wavenumber domain $\left(x, K_{y}, z\right)$

$$
-\nabla \cdot\left(\sigma(x, z) \nabla \bar{\phi}\left(x, K_{y}, z\right)\right)+K_{y}^{2} \sigma(x, y) \bar{\phi}\left(x, K_{y}, z\right)=Q \delta\left(x_{s}\right) \delta\left(z_{s}\right)
$$

where $K_{y}$ is the wavenumber in the $y$ direction, $\bar{\phi}$ is the transformed potential and $Q$ is the constant steady state current density (Dey and Morrison, 1979). The relationship between the transformed and the normal potential is a cosine Fourier transform

$$
\begin{gathered}
\bar{\phi}\left(x, K_{y}, z\right)=\int_{0}^{\infty} \phi(x, y, z) \cos \left(K_{y} y\right) d y \\
\phi(x, y, z)=\frac{2}{\pi} \int_{0}^{\infty} \bar{\phi}\left(x, K_{y}, z\right) \cos \left(K_{y} y\right) d K_{y} .
\end{gathered}
$$

Due to the reciprocity theorem and the $2 \mathrm{D}$ resistivity distribution, the integral of the potential along $y$ axis $\int_{-\infty}^{\infty} \phi\left(x_{r}, y, z_{r}\right) d y$ due to a point source with current intensity $I_{s}$ at $\left(x_{s}, y_{s}, z_{s}\right)$, equals to the potential $\phi\left(x_{r}, y_{s}, z_{r}\right)$ due to a line source extended at $y$ dimension at $\left(x_{s}, z_{s}\right)$ with current intensity per meter equals to $I_{s}$. Consequently, the potential $\Phi$ due to a line source at $\left(x_{s}, z_{s}\right)$ with current intensity per meter $Q$, equals to

$$
\Phi(x, z)=\int_{-\infty}^{\infty} \phi(x, y, z) d y=2 \bar{\phi}(x, 0, z) .
$$

Substituting (5) to (2) results to

$$
-\nabla \cdot(\sigma(x, z) \nabla \Phi(x, z))=2 \bar{Q} \delta\left(x_{s}\right) \delta\left(z_{s}\right)
$$

Equation (6) is the partial differential equation that describes the spatial distribution of the electric potential due to a line source located at $\left(x_{s}, z_{s}\right)$ with current intensity per meter equals $\bar{Q}$. The relationships between $\Phi \rightarrow \bar{\phi}$ and $\bar{\phi} \rightarrow \phi$ are exploited in Appendix A in order to derive analytical solutions for homogeneous space and two-layered media. The 
solution for homogeneous half-space is essential for numerically evaluating (6) in a grid-interdependent manner (Spitzer et. al , 1999). The analytical solution for two-layered media is used as a benchmark to evaluate the suggested numerical framework (see Appendix A), especially in cases when high resistivity contrasts are present e.g. marine surveys (see chapter 4.3).

\section{1 | Grid-independent finite-difference scheme}

While numerical algorithms for solving partial differential equation (finite differences, finite elements etc.) depend on grid geometry, Spitzer et. al (1999) suggested a method in which Poisson's equation is numerically evaluated for grid-independent electrode positioning. This is particularly important for any optimization scheme meant to estimate the coordinates of a sheet-like body. Having the ability to map the optimization space in a continuous manner facilitates interpretation by avoiding integer-based gradients and integer-steps in convex and global optimizers respectively. In addition, a grid-independent based inversion can estimate the position of a dipole accurately without the usage of dense grids or dense sub-grids within a sparse main grid like the methodology proposed by Haas et. al. (2013).

Assuming a homogeneous half-space, equation 6 becomes

$$
-\bar{\sigma} \nabla^{2} \Phi_{n}(x, y)=2 \bar{Q} \delta(x) \delta(z)
$$

where $\bar{\sigma}$ is the homogeneous resistivity distribution and $\Phi_{n}$ is the potential measured subject to a homogeneous half-space. Substituting 7 to 6 results to

$$
\nabla \cdot(\sigma(x, z) \nabla \Phi(x, y))=\bar{\sigma} \nabla^{2} \Phi_{n}(x, z)
$$

The potential $\Phi$ can be divided into an anomalous $\left(\Phi_{a}\right)$ and normal $\left(\Phi_{n}\right)$ part

$$
\Phi=\Phi_{a}+\Phi_{n}
$$

From (9) and (8) it is easy to show that

$$
\nabla \cdot\left(\sigma(x, z) \nabla \Phi_{a}(x, y)\right)=-\nabla \cdot\left((\sigma(x, z)-\bar{\sigma}) \nabla \Phi_{n}(x, z)\right)
$$

Equation 10 can be written in a discretized form as

$$
\mathbf{K}_{a} \mathbf{V}_{a}=\mathbf{K}_{n} \mathbf{V}_{n}
$$

where $\mathbf{K}_{a}$ is the stiffness matrix for $\sigma, \mathbf{K}_{n}$ is the stiffness matrix for $\bar{\sigma}-\sigma, \mathbf{V}_{a}$ is a vector containing the anomalous potential and $\mathbf{V}_{n}$ is a vector containing the potential for a homogeneous half-space. The anomalous potential $\mathbf{V}_{s}$ is to be found since both stiffness matrices and $\mathbf{V}_{n}$ (see Appendix $\mathrm{A}$ ) are known. The vector $\mathbf{V}_{n}$ can be evaluated for any arbitrary source position since it has a known analytical expression (23). From (11) it is clear that the load vector is substituted by the vector $\mathbf{K}_{n} \mathbf{V}_{n}$. The latter is an apparent load vector, from which it is possible to obtain the values of the anomalous part of the potential for any arbitrarily placed sheet-like body. Subsequently, the anomalous part of the potential $\Phi_{a}$ is added to $\Phi_{n}$ in order to obtain $\Phi$. Notice that in an optimization scheme, the stiffness matrices $\mathbf{K}_{a}$ and $\mathbf{K}_{n}$ have to be evaluated one time since in each iteration only the vector $\mathbf{V}_{n}$ is changing. 
The stiffness matrices are calculated using the integral method suggested by Dey and Morrison (1979) for $K_{y}=0$. Regarding the boundaries, the Newman boundary condition is chosen in order to avoid instabilities arising from the fact that line sources can not converge to zero at the boundaries (Nostrand and Kenneth, 1966). The Newman boundary condition constrains the normal spatial derivatives to be zero at the boundaries (Dey and Morrison, 1979). Thus, the resulting fields are relative to a reference point that has to be defined by the user. This is the equivalent of the fixed electrode measurements that often applied in the field.

\section{3 | HYBRID PSO-LINEAR OPTIMIZATION}

Particle-swarm optimization (PSO) is a biological based global optimizer that mimics the behavior of swarms in nature (Robinson and Rahmat-Samii , 2004). PSO has proven very effective in optimizing multi-dimensional problems and in certain cases outperforms other global optimizers like genetic algorithms (Kennedy and Spears, 1998). PSO is relatively new in geophysics (Shaw and Srivastava , 2007) and has been successfully applied to seismics (Haijun et. al , 2017), 1D-DC (Martinez et. al , 2010), gravity (Pallero et. al , 2015, 2017) and SP (Gokturkler and Balkaya, 2012; Peksen et. al. , 2011; Santos , 2010). In the present paper, PSO is chosen in an effort to overcome local minimal that are present in complex resistivity environments and when multiple sheet-like bodies are meant to be recovered. In addition, we apply a hybrid scheme that combines PSO with least-squares (Kelley et. al , 2007). The latter is proven to have substantially higher convergence rate compared to simple PSO.

PSO uses the concept of moving particles inside a predefined optimization space. Similar to a swarm of insects moving in a 3D space, the particles in PSO are moving in a multi-dimensional space searching for the coordinates in which the given error function is minimized. PSO is initialized by choosing the number of particles and defining the upper and lower bounds of the problem. The particles are constrained to move within these bounds avoiding unnecessary searches for unrealistic scenarios. The particles are randomly placed within the predefined domain and for each particle a cost function is evaluated. Subsequently an initial velocity is chosen for each particle in a random manner. In every iteration each particle remembers its best performance and its corresponding n-dimensional coordinates. In addition, the best performance of the whole swarm is also saved along with its corresponding n-dimensional coordinates. Based on these, the velocity vector is updated according to (Shi and Eberhart, , 1998)

$$
v_{n}^{\tau}=w \cdot v_{n}^{\tau-1}+c_{1} \cdot \operatorname{rand}()\left(q_{n}^{\tau-1}-q_{n}^{b}\right)+c_{2} \cdot \operatorname{rand}()\left(q_{n}^{\tau-1}-Q_{b}\right)
$$

where $v_{n}^{\tau}$ and $q_{n}^{\tau}$ are the velocity (parameter correction vector) and the position (parameter) of the $n$th particle at $\tau$ iteration respectively, $q_{n}^{b}$ is the best position for the particle $n$ and $Q_{b}$ is the best position for all the particles in the swarm. The variables $w, c_{1}$, and $c_{2}$ are constants that adjust the behavior of the swarm. Equation 12 is a stochastic relationship with rand () being a uniform distribution between $[0,1]$ (Kelley et. al , 2007). Notice that for each particle the velocity is a vector with dimensions equal to the number of the parameters need to be optimized. The velocity vector is used to update the particle's position based on a simple step procedure $q_{n}^{\tau+1}=q_{n}^{\tau}+v_{n}^{\tau}$.

The first term of (12) forces the particle to continue its previous trajectory allowing it to explore "worse" solutions thus overcoming local minimal. The second parameter makes each particle biased to directions towards $q_{n}^{b}$, i.e. the position in which every particle independently had its best performance. Lastly, the third term shifts the whole swarm towards the position in which the best swarm-performance is achieved. In every iteration, the positions of the particles are updated according to the estimated velocities using (12). The procedure is repeated for a predefined number of iterations or when some given convergence criteria are met. Large values for $c_{2}$ results to faster convergence with the 
expense of increasing the chances of getting trap to local minimal. Large $w$ reduces the chances of getting trap to local minimal with the expense of decreasing the convergence rate. Choosing the optimized parameters $w, c_{1}$ and $c_{2}$ that balance between efficiency and accuracy is case-dependent and various values have been proposed in the literature (Robinson and Rahmat-Samii , 2004). Although generic PSO parameters applicable to any type of problem are very appealing, nonetheless, as it is stated by (Dieterich and Hartke, 2000), a specific version tuned to the problem at hand is a more suitable choice. In addition, generic PSO parameters are tuned based on clinical functions (spherical functions, Schaffer's $f 6$ function etc.) that often deviate from reality leading to false conclusions regarding the performance of PSO (Dieterich and Hartke, 2000). For the current problem, the parameters should be set such as for PSO to effectively convergence to a global solution for a diverse set of scenarios. From simple cases such as a single dipole buried in a homogenous half-space to multiple dipoles subject to a complex stochastic resistivity distribution. In the first scenario, $w=0$ and $c_{1}=c_{2}=1$ will result to a fast convergence rate since the problem lacks of local minimal when proper initialization is applied. For the second case, a large $w$ is necessary in order to tackle the numerous local minimal and plateaus present in the problem due to the multiple sheet-like bodies and the complex resistivity distribution.Through trial and error, and in an effort to be conservative, $w=1, c_{1}=1$ and $c_{2}=0.5$ are chosen for the current problem. The number of particles used in PSO is also crucial. Using small number of particles increases the efficiency of the algorithm by compromising accuracy. A number between $40-70$ is proven sufficient for the examples examined in this study.

The optimization space for multiple sheet-like bodies is $\mathbb{R}^{5 S}$ where $S$ is the number of sheet-like bodies. In particular, every sheet-like body is a dipole for which its coordinates and current intensity per meter are to be found. The hybrid scheme that it is used in the present study takes advantage of the fact that parts of the unknowns can be expressed with respect to others in a linear manner. To further elaborate on that, we introduce the function $E(\mathbf{x}, \mathbf{I})$ where $\mathbf{x}$ and $I$ are both vectors needed to be fine-tuned in order to optimize $E$. The hybrid scheme can be applied only if $I$ can be expressed with respect to $x$ in a linear manner. If the optimized $I$ for every set of $x$ can be evaluated using linear least squares then the function can be re-written as $E(\mathbf{x}, W(\mathbf{x}))$. The hybrid scheme can be applied using both global optimizers and convex non-linear inversion. The non-linear optimization will search in the optimization space defined by $\mathbf{x}$ since the optimized $\mathbf{I}$ can be expressed with respect to any set of $\mathbf{x}$ using linear least-squares. This reduces the optimization space and accelerates the convergence rate to problems that can be expressed in this form (Xu et. al , 2000; Kelley et. al, 2007).

From (11) it is evident that changing the current intensity per meter affect only the vector $V_{n}$ in a linear manner. Consequently, by setting the absolute value of the current intensity per meter to one, the problem can be stated as

$$
\mathbf{M}=\mathbf{G l}
$$

where $\mathbf{M}$ is a vector that contains the measurements and $\mathbf{G}=\left[\mathbf{V}_{1}, \mathbf{V}_{2} \ldots \mathbf{V}_{S}\right]$ is a matrix that contains the synthetic measurements for each sheet-like body setting their current intensity per meter to one. The optimized current intensity per meter for every sheet-like body can be evaluated using least squares

$$
\mathbf{I}_{L S Q}=\left(\mathbf{G}^{T} \mathbf{G}\right)^{-1} \mathbf{G}^{T} \mathbf{M}
$$

where $\mathbf{I}_{L S Q}=\left[I_{1}, I_{2} \ldots I_{S}\right]^{T}$ is a vector that contains the current intensity per meter for each sheet-like body. Substituting 14 to 13 , the synthetic data can be expressed only as a function of the coordinates of the sheet-like bodies

$$
\mathbf{M}_{s y n}=\mathbf{G}\left(\mathbf{G}^{T} \mathbf{G}\right)^{-1} \mathbf{G}^{T} \mathbf{M}
$$


Notice that by expressing the current intensity of a sheet-like body with respect to its coordinates the optimization space is reduced since the cost function needed to be optimized is only related to the coordinates of the sheet-like bodies

$$
\text { Cost }=\frac{\left\|\mathbf{M}-\mathbf{M}_{\text {syn }}\right\|^{2}}{N}=\frac{\left(\mathbf{M}-\mathbf{G}\left(\mathbf{G}^{T} \mathbf{G}\right)^{-1} \mathbf{G}^{T} \mathbf{M}\right)^{T}\left(\mathbf{M}-\mathbf{G}\left(\mathbf{G}^{T} \mathbf{G}\right)^{-1} \mathbf{G}^{T} \mathbf{M}\right)}{N}
$$

where $N$ is the number of the measurements. The hybrid scheme employed in the present paper uses PSO to minimize equation (16). Nonetheless, other global optimizers like genetic algorithms or modified non-linear schemes (Soupios et. al , 2011) can be applied instead of PSO in a straightforward manner. To illustrate the effect of the hybrid scheme to the overall performance of the optimization, both the PSO and the proposed hybrid scheme are applied in two cases studies. The first case consists of a two layered medium with $\rho_{1}=50$ ohm.m, $\rho_{2}=100$ ohm.m and $h=10 \mathrm{~m}$. A single dipole is placed in the first layer and its current intensity equals to $I=-3 \mathrm{~mA}$. In the second case, $\rho_{1}=300 \mathrm{ohm} . \mathrm{m}, \rho_{2}=100 \mathrm{ohm} . \mathrm{m}$ and $h=20 \mathrm{~m}$. Two sheet-like bodies are placed within the first layer with different polarizations with current intensity equals to $I=3 \mathrm{~mA}$. Figure 1 shows the cost function (16) for every iteration using the hybrid scheme and the PSO. Both methods use 40 particles and the same $w, c 1$ and $c 2$. It is apparent that the proposed method convergences much faster than traditional PSO which facilitates interpretation. Notice that as the number of dipoles increases, the performance of traditional PSO is reduced compared to the hybrid scheme. This makes the proposed methodology crucial for interpreting multiple sheet-like bodies in an efficient manner.

\section{4 | NUMERICAL EXPERIMENTS}

In the current section, four numerical case studies are investigated using the hybrid PSO optimization. Two examples of surface measurements are given, one with surface-borehole measurements and one example coming from marine geophysics. The number of particles is set to 50 and the parameters of PSO are $w=1, c 1=1$ and $c 2=0.5$. For all four cases, the hybrid scheme converges in less than 60 iterations. The following examples use the same scale for convenience. It is apparent, that based on the application, the problem can be re-scaled accordingly in a straightforward manner.

The following examples illustrate the potential of the proposed method to estimate the coordinates of sheet-like bodies. It should be noticed that the accuracy of the hybrid scheme can be compromised from noise, non-adequate measurements and the accuracy of the estimated resistivity distribution. Nonetheless, using adequate measurements and a good resistivity approximation, the current technique can approximate the coordinates of multiple sheet-like bodies in an efficient manner. Recovering the current intensity per meter is very challenging and under realistic circumstances the proposed method is not capable of accurately recovering it. The reason for this is highlighted in the analytical solutions for homogeneous half-space and two-layered media. From (23), (26) and (27) it is evident that resistivity and current intensity per meter consist a non-unique set. Thus, discrepancies between the estimated and the actual resistivity distribution greatly affect the estimated current intensity per meter.

\section{1 | Ground survey}

In the first example, a simple geometry excited by a single sheet-like body is examined. The model is a two-layer structure with a resistive bedrock ( $500 \mathrm{o.ohm}$ ) and a conductive overburden ( $40 \mathrm{ohm} . \mathrm{m}$ ). A step-like feature is implemented in an effort to simulate a typical geometry associated with normal faults. The sheet-like body is placed underneath the step 
of the bedrock. Figure 2 illustrates the simulated geometry as well as the inversion results. Notice that a smoothed resistivity is used as background for the hybrid scheme in order to simulate the resulting resistivity distribution using ERT (Constable et. al , 1987). Low level Gaussian noise $(2-3 \mathrm{mV}$ ) is also added to the data. Similar noise levels are added to all the numerical examples in this section in an effort to simulate cases with good overall signal to noise ratio. In hydro-geological applications in which the signal is usually low (Minsley, 2007) the noise is expected to be more dominant and pre-processing should be considered. The resulting sheet-like body using the suggested hybrid scheme is in good agreement with the actual model. This showcases the capability of the proposed framework at detecting single faults in complex resistivity distributions.

The case study above has mostly theoretical significance since faults and fractures usually occur in groups resulting from the stresses applied to a given area. Thus, it is crucial for an algorithm to be capable to interpret SP anomalies resulting from multiple sheet-like bodies in an efficient manner. The second example investigates the case of conjugate faults and the importance of choosing the right number of sheet-like bodies is highlighted. The investigated model is shown in Figure 3. The resistivity of the bedrock and the overburden layer are $200 \mathrm{ohm} . \mathrm{m}$ and $70 \mathrm{ohm}$.m respectively. The sheet-like bodies are placed beneath the fault lines (see Figure 3). Similar to the previous example, a smoothed edition of the actual resistivity model is used during the inversion and Gaussian noise is added to the observed data. In Figure 3 the results using one and two sheet-like bodies are shown. It is apparent that using one sheet-like body does not adequately fit the measurements. Using two sheet-like bodies results to a stable and accurate solution that sufficiently approximates the actual model. Increasing the number of sheet-like bodies increases the non-uniqueness which consequently results to multiple solutions with the same accuracy. This is highlighted in Figure. 4 in which three sheet-like bodies are used during the inversion. Due to the stochastic nature of PSO, in the presence of non-uniqueness, the algorithm will converge to different results with each execution. In Figure 4 three different solutions are illustrated that result to the same potential distribution on the surface. Thus, increasing the number of sheet-like bodies should be followed by an increase of the available information either through extra measurements (longer measurement lines, borehole data etc.) or through constrains coming from geological or geophysical data.

\section{2 | Surface-Borehole survey}

In the third example, four sheet-like bodies are distributed in a realistic resistivity distribution that is generated using fractal correlated noise (Turcotte , 1992). Due to the inherited non-uniqueness of SP, surface measurements are not adequate to resolve multiple sheet-like bodies concentrated in a relative small space (see previous example). Consequently, both surface and borehole measurements are employed in the current example in an effort to increase the available information. Figure 5 shows the positions of the boreholes as well as the positions of the sheet-like bodies. Similar to the previous examples, a smoothed edition of the original resistivity distribution is used during the inversion. Figure 5 illustrates the results using the proposed hybrid inversion. The estimated sheet-like bodies are in good agreement with the actual ones indicating that the proposed scheme, when adequate information is available, can be used for estimating multiple sheet-like bodies in complex resistivity distributions.

\section{3 | Marine survey}

$\mathrm{SP}$ is an attractive technique for detecting marine ore deposits associated with geothermal activity (Kawada and Kasaya , 2017). The reasons for that are the reduced noise levels and the ability to contact continuous measurements (Kawada and Kasaya , 2017). The biggest obstacle in marine SP surveys is the fact that water mass is a very conductive medium that acts as a low pass filter, thus reducing the available information. Nonetheless, measurements can be taken at 
different depths increasing the available information and the overall reliability of the interpretation.

The case study examined here is illustrated in Figure 6. Two faults are placed inside a bedrock with a stochastically varying resistivity. The average resistivity of the bedrock is $\approx 150 \mathrm{ohm} . \mathrm{m}$ and the resistivity of the water mass is 1 ohm.m. Three different profile measurements are taken. Profile-A follows the topography of the bedrock. Profile-B is a straight line at $20 \mathrm{~m}$ depth. And Profile- $\mathrm{C}$ is a straight line at $10 \mathrm{~m}$ depth. All three lines are used as inputs to the hybrid scheme. Similar to the previous examples, a smoothed resistivity distribution is used during the inversion and the observed data are corrupted with Gaussian noise. Figure 6 illustrates the resulting sheet-like bodies using the proposed scheme. The edges of the sheet-like bodies are adequately approximated. The accuracy of the prediction is related to the depth of the target. This is because deeper poles have a small contribution to the overall signal, thus they are very susceptible to noise and discrepancies between the simulated and the actual resistivity distribution.

\subsection{Uncertainty Analysis}

The current section evaluates the sensitivity of the current technique to noise and clutter. We define as noise everything that can be described effectively as a Gaussian process. This includes errors in the positioning of the electrodes as well as the inherited noise of the system. The term "clutter" is usually used to characterize a more systematic source of errors. Here, clutter is chosen to describe the errors that occur due to the discrepancies between the actual and the estimated resistivity distribution. The effect of clutter has been investigated for extreme cases (Minsley, 2007; Giannakis et. al , 2012), where major simplifications (homogeneity assumption) take place mis-representing the actual model completely. Here we examine more realistic scenarios, in which the resistivity distribution is a smooth edition of the actual one.

The case study examined in this section is illustrated in Fig. 7. A single dipole is placed inside a fractal resistivity distribution. The resistivity varies from 30-330 ohm.m. The resulting signal is corrupted with Gaussian noise with zero mean and an increasing standard deviation (2.5, 5 and $10 \mathrm{mV}$ ). For each noise level the hybrid scheme is applied and the results are shown in Fig. 8. It is evident that when adequate measurements are taken (i.e. when the measurement step is adequately small) the proposed methodology can effectively deal with Gaussian noise even in the case of highly corrupted data.

Fig. 9 shows the inversion results using a smoothed edition of the actual resistivity distribution. The highwavenumber components of the model are gradually reduced in an effort to introduce clutter to the model. Although incorporating the resistivity distribution is crucial (Minsley, 2007; Giannakis et. al , 2012), nonetheless a first level approximation is proven to be adequate for accurately recovering the coordinates of the dipole using the proposed framework (see Fig. 9).

Lastly we want to evaluate the ability of the proposed approach to perform under sparse sets of data. To that extend, different measurement-steps are used starting from dense configurations to sparse ones. The data are corrupted with Gaussian noise with $2.5 \mathrm{mV}$ standard deviation and the resistivity model used during the inversion is the actual one (non-smoothed version). As it is shown in Fig. 10, for a reasonable measurement-step and noise-level, the suggested methodology manage to accurately recover the position of the sheet-like body.

\section{5 | REAL MEASUREMENTS}

In the current section, the proposed technique is applied on real data acquired during a geophysical investigation that took place in Chalkidiki, northern Greece (Vargemezis et. al , 2012). The investigated area consists primarily 
of ophiolithic outcrops. Prior to the investigations, there were geological indications that the contact between the ophiolites and the overburden sediments is associated with a possible fault that lead to the subduction of the ophiolites (Vargemezis et. al , 2012). A combined focusing approach using ERT, SP and very low frequency (VLF) electromagnetic method was applied (Vargemezis et. al , 2012) in order to locate possible water zones associated with the suspected faults. Subsequently, a borehole was drilled that confirmed the geophysical results. For more details regarding the geology of the area as well as the results of the geophysical investigation the reader is referred to Vargemezis et. al (2012).

Here we focus on the tomography line in which the borehole was drilled. The tomography line is 235 meters long and the resistivity distribution is mapped down to 60 meters depth. The surface is relatively smooth and for the current inversion it is assumed to be flat. The ERT profile consists of 48 electrodes with 5 meters spacing. Both dipole-dipole and pole-dipole measurements were used and the resulting resistivity distribution is shown in Figure 11 (Vargemezis et. al, 2012). A low resistivity zone can be observed at $x, z \approx 150,50$. VLF data also suggest a low resistivity zone at that area associated with a normal fault (Vargemezis et. al , 2012). Following the geophysical indications, a borehole was drilled at $x \approx 150$ meter (see Figure 11) that confirmed the geophysical results and led to a water supply of $5-10 \mathrm{~m}^{3} / \mathrm{h}$.

Using the resulting resistivity tomography and the SP data provided by Vargemezis et. al (2012), the proposed hybrid scheme is applied assuming two unknown sheet-like bodies. The results are shown in Figure 11. One normal fault is recovered at around $x \approx 160$ meters. A normal fault at that position is in good agreement with all the available geophysical evidences as well as the borehole outcomes. In addition to the normal fault, another deeper fault is recovered (see Figure 11) with much smaller current intensity per meter compared to the normal fault. Although there are some evidences that point to the existence of the second fault (Vargemezis et. al , 2012), nonetheless further geophysical investigations are needed for a conclusive result. Due to its small current intensity per meter (compared to the normal fault) and its large depth, the second fault might be an artifact that addresses the non-optimal background-removal (Jagannadha et. al , 1993) of the SP data prior to the inversion (see Figure 11).

\section{6 | CONCLUSIONS}

A grid-independent finite-difference scheme is used in order to implement multiple sheet-like bodies in arbitrary locations subject to a given $2 \mathrm{D}$ resistivity distribution. The proposed framework is validated using novel analytical solutions for 2D dipoles in two-layered media. The ability to excite the model with grid-independent sources allow us to use non-linear optimization and map the optimization space in a continuous manner. In the present work, a hybrid PSO-linear optimization is suggested that manages to overcome local minimal that are present in realistic resistivity distributions. It is shown that the proposed hybrid scheme converges substantially faster compared to typical PSO while retaining its accuracy. Through numerical and real experiments, strong indications are given to support the premise that the suggested technique can be a useful asset at detecting geological targets associated with faults i.e. marine mineral deposits, hydrothermal ore deposits and so on. Lastly, it should be emphasized that the proposed methodology can be seen as a dipole-based inversion in which the user constrains the number of dipoles while their intensity is expressed with respect to their coordinates. Consequently, the hybrid optimization scheme can be applied in a similar manner to other potential-field methods by allowing the user to define the number of gravitational or magnetic poles based on the available information. 


\section{A I ANALYTICAL SOLUTION FOR TWO LAYERED MEDIA}

Analytical solutions for sheet-like bodies in homogeneous half-spaces have been widely used in the SP literature (Meiser , 1962; Atchuta, 1982; Akgun , 2001). Nonetheless, analytical solutions for line sources embedded in two-layered media -to our knowledge- have never been reported. The latter are important when numerical solvers need to be tested in a challenging scenario. In addition, when the geology of an area can be represented by a two-layered structure, an analytical solution can provide a practical and elegant interpretation tool avoiding computationaly intense numerical approaches.

The relationship between $\phi(x, y, z) \rightarrow \bar{\phi}(x, 0, z) \rightarrow \Phi(x, z)$, described in section 2.1, can be exploited in order to use known analytical solutions for $\phi$ and transformed them to $\Phi$. For homogeneous half space the surface potential due to a point source located at the origins of the axes $(0,0,0)$ equals to (Nostrand and Kenneth, 1966)

$$
\phi(x, y, z)=\frac{I \rho}{2 \pi} \frac{1}{\sqrt{x^{2}+y^{2}+z^{2}}}
$$

where $I$ is the current intensity of the point source and $\rho$ is the resistivity $(1 / \sigma)$. Substituting (17) to (3) results to

$$
\bar{\phi}\left(x, K_{y}, z\right)=\frac{I \rho}{2 \pi} \int_{0}^{\infty} \frac{\cos \left(K_{y} y\right)}{\sqrt{x^{2}+y^{2}+z^{2}}} d y=\frac{I \rho}{2 \pi} K_{0}\left(K_{y} \sqrt{x^{2}+z^{2}}\right)
$$

where $K_{0}$ is the zero-order modified Bessel function of the second kind (Xu et. al , 2000). Substituting (18) to (5) results to

$$
\Phi(x, z)=\frac{I \rho}{\pi} \lim _{K_{y} \rightarrow 0} K_{0}\left(K_{y} \sqrt{x^{2}+z^{2}}\right) .
$$

The potential is an artificial non-physical parameter which is related to the electric field through its gradient $E=-\nabla \phi$. Thus, the boundaries of the potential field are arbitrarily set and often assumed to be zero at infinity. Equation 19 equals to infinity for any set of coordinates. Thus, the potential due to a line source can not be expressed as a function that converges to a given value at infinity. Nostrand and Kenneth (1966) initially mention this unique aspect of line sources. Subsequently they explained that the potential due to a line source can be evaluated only with respect to a reference point since zero-value (or any value) boundary conditions can not be implemented. This is not the case when linear dipoles are used to excite the model. Using two equal sources with opposite signs results to

$$
\Phi(x, z)=\frac{I \rho}{\pi} \lim _{K_{y} \rightarrow 0}\left(K_{0}\left(K_{y} \sqrt{x_{1}^{2}+z_{1}^{2}}\right)-K_{0}\left(K_{y} \sqrt{x_{2}^{2}+z_{2}^{2}}\right)\right) .
$$

Where $\left(x_{1}, z_{1}\right)$ and $\left(x_{2}, z_{2}\right)$ are the coordinates with respect to the measurement point of the positive and negative sources respectively (see Fig. 12). When $K_{y}$ tends to zero the zero-order modified Bessel function of the second kind can be expressed as (Abramowitz and Stegun , 1964; Arfkens and Weber , 2001)

$$
\lim _{K_{y} \rightarrow 0} K_{0}\left(K_{y} r\right)=-\lim _{K_{y} \rightarrow 0}\left(\ln \left(\frac{K_{y} r}{2}\right)\right)+\gamma
$$


where $\gamma$ is the Euler-Mascheroni constant (Abramowitz and Stegun, 1964). Substituting (21) to (20) results to

$$
\Phi(x, z)=\frac{I \rho}{\pi} \lim _{K_{y} \rightarrow 0}\left(\ln \left(\frac{K_{y} \sqrt{x_{2}^{2}+z_{2}^{2}}}{2}\right)-\ln \left(\frac{K_{y} \sqrt{x_{1}^{2}+z_{1}^{2}}}{2}\right)\right) .
$$

Further expanding the logarithms leads to

$$
\Phi(x, z)=\frac{I \rho}{\pi}\left(\ln \sqrt{x_{2}^{2}+z_{2}^{2}}-\ln \sqrt{x_{1}^{2}+z_{1}^{2}}\right)=\frac{I \rho}{\pi} \ln \left(\frac{r_{2}}{r_{1}}\right)
$$

where $r_{1}=\sqrt{x_{1}^{2}+z_{1}^{2}}$ and $r_{2}=\sqrt{x_{2}^{2}+z_{2}^{2}}$. Equation 23 is the well known equation that describes the surface potential due to a sheet-like body with edges at $\left(x_{1}, z_{1}\right)$ and $\left(x_{2}, z_{2}\right)$ respectively (Jagannadha et. al, 1993).

The same procedure i.e. transforming $\phi \rightarrow \Phi$, can be applied to derive the analytical solution of line sources subject to two-layered media. The surface potential due to a point source in a two layered medium equals to (Tang et. al , 2011)

$$
\begin{gathered}
\phi(x, y, z)=\frac{I \rho_{1}}{2 \pi}\left[\frac{1}{\sqrt{x^{2}+y^{2}+z^{2}}}+\sum_{n=1}^{\infty} k^{n}\left(\frac{1}{\sqrt{x^{2}+y^{2}+(2 n h-z)^{2}}}+\frac{1}{\sqrt{x^{2}+y^{2}+(2 n h+z)^{2}}}\right)\right], \quad z \leq h \\
\phi(x, y, z)=\frac{I \rho_{1}(1+k)}{2 \pi} \sum_{n=1}^{\infty} k^{n}\left(\frac{1}{\sqrt{x^{2}+y^{2}+(2 n h+z)^{2}}}\right), \quad z \geq h
\end{gathered}
$$

where $h$ is the depth of the first layer, $k=\frac{\rho_{2}-\rho_{1}}{\rho_{2}+\rho_{1}}$ and $\rho_{2}, \rho_{1}$ are the resistivities of the second and first layer respectively. Transforming $\phi \rightarrow \Phi$ similar to the homogeneous case and using dipoles to enforce zero-valued boundary condition results to

$$
\begin{gathered}
\Phi(x, z)=\frac{I \rho_{1}}{\pi}\left(\ln r_{2}-\ln r_{1}+\sum_{n=1}^{\infty} k^{n}\left(A_{n}+B_{n}\right)\right), \quad z_{1}, z_{2} \leq h \\
\Phi(x, z)=\frac{I \rho_{1}(1+k)}{\pi} \sum_{n=1}^{\infty} k^{n} C_{n}, \quad z_{1}, z_{2} \geq h \\
A_{n}=\ln \sqrt{x_{2}^{2}+\left(2 n h-z_{2}\right)^{2}}-\ln \sqrt{x_{1}^{2}+\left(2 n h+z_{1}\right)^{2}} \\
B_{n}=\ln \sqrt{x_{2}^{2}+\left(2 n h+z_{2}\right)^{2}}-\ln \sqrt{x_{1}^{2}+\left(2 n h-z_{1}\right)^{2}} \\
C_{n}=\ln \sqrt{x_{2}^{2}+\left(2 n h+z_{2}\right)^{2}}-\ln \sqrt{x_{1}^{2}+\left(2 n h+z_{1}\right)^{2}}
\end{gathered}
$$


To validate the accuracy of the grid-independent numerical scheme presented in 2.1, we compare the analytical and numerical solutions for two case studies (see Figure 13). In the first example the resistivity of the overburden layer is $50 \mathrm{ohm}-\mathrm{m}$ and that of the bedrock is $400 \mathrm{ohm}-\mathrm{m}$. The thickness of the first layer is $50 \mathrm{~m}$. The sheet-like body is placed in a non-integer position inside the first layer. The second case consists of two layers with $30 \mathrm{ohm} . \mathrm{m}$ and $300 \mathrm{ohm} . \mathrm{m}$ respectively. The first layer has $10 \mathrm{~m}$ thickness. The sheet-like body is tilted and placed within the bedrock. Similar to the first example the fault is placed in a non-grid position. Figure 13 shows that the numerical and the analytical results are in good agreement indicating the validity of the grid-independent FD scheme. Before we apply the hybrid scheme to marine surveys (see chapter 4.3), the grid-independent numerical framework should be validated in environments with high resistivity contrasts. Figure 14 illustrates the comparison between analytical and numerical results for two layered media with varying resistivity contrast. It is evident that the suggested scheme can be reliably used to simulate marine environments in which a conductive water mass lays on top of a resistive bedrock. 


\section{LIST OF FIGURES}

1 Convergence rate of the hybrid PSO compared to simple PSO. Up: The model is a two layered medium with $\rho_{1}=50$ ohm.m, $\rho_{2}=100 \mathrm{ohm} . \mathrm{m}$ and $h=10 \mathrm{~m}$. A tilted sheet-like body is placed in the first layer with current intensity $I=-3 \mathrm{~mA}$. Down: The model is a two layered media with $\rho_{1}=300 \mathrm{ohm} \cdot \mathrm{m}, \rho_{2}=100 \mathrm{ohm} \cdot \mathrm{m}$ and $h=20 \mathrm{~m}$. Two normal to the surface sheet-like bodies are placed parallel to each other with $10 \mathrm{~m}$ distance. In both examples the parameters of PSO are, $w=1, c 1=1, c 2=0.5$ and the number of particles equals to $40 \ldots \ldots \ldots$

2 Down: The self-potential anomaly generated by a normal fault. The resistivity of the bedrock and the overburden layer is $500 \mathrm{ohm} . \mathrm{m}$ and $40 \mathrm{ohm} . \mathrm{m}$ respectively. Up: The resulting sheet-like body using the proposed scheme subject to a smoothed resistivity distribution. The observed data are illustrated with circles while the calculated SP

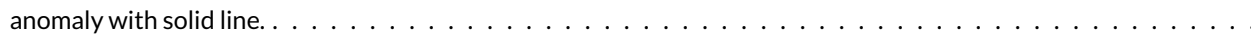

3 C: The self-potential anomaly generated by two normal faults. The resistivity of the bedrock and the overburden layer is $200 \mathrm{ohm} . \mathrm{m}$ and $70 \mathrm{ohm} . \mathrm{m}$ respectively. B: The resulting sheet-like body using the proposed scheme subject to a smoothed resistivity distribution. The observed data are illustrated with circles while the calculated SP

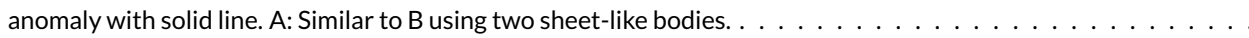

4 The observed and the calculated SP anomaly are illustrated with circles and solid lines respectively. The model is similar to the one described in Figure 3. Three different cases are shown that sufficiently fit the measured data. This highlights the fact that inverting for multiple sheet-like bodies requires adequate information (data or constrains) in order to tackle the non-uniqueness embedded in SP measurements.

5 Left Down: The self-potential anomaly generated by four dipoles. A fractal correlated noise is used to generate a realistic resistivity distribution. Left Up: The resulting sheet-like bodies using the proposed scheme subject to a smoothed resistivity distribution. On the right, the observed and calculated data are illustrated with circles and

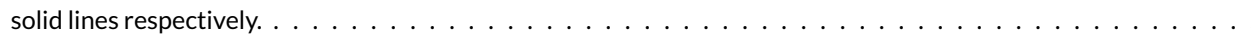

6 Left Down: The self-potential anomaly generated by two dipoles in a marine environment. The water mass is illustrated with light blue colour. The resistivity of the water is $1 \mathrm{ohm} . \mathrm{m}$. Left Up: The resulting sheet-like bodies using the proposed scheme subject to a smoothed resistivity distribution. On the right, the observed and calculated data are illustrated with circles and solid lines respectively. . . . . . . . . . . . . . . . . . .

7 The actual model used to evaluate the sensitivity of the current technique to noise and clutter. A single tilted dipole is placed inside a fractal resistivity distribution which varies from $30-330 \mathrm{ohm} . \mathrm{m}$. The measurement-step equals to

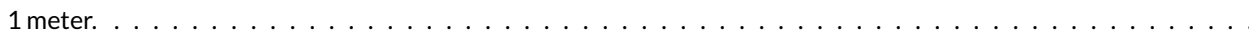

8 The hybrid scheme is applied to data with different levels of noise. The inverted dipole is illustrated with red line. The actual position of the dipole is shown in Fig. 7. The data are corrupted with Gaussian noise with 2.5,5 and $10 \mathrm{mV}$ standard deviation. It is evident that the proposed scheme can affectively deal with Gaussian noise (in the presence of dense measurements) and accurately recover the coordinates of the sheet-like body. Due to the sufficient number of measurements, the squared root of the misfit function (16) is close to the standard deviation of the noise, in particular $\sqrt{\text { Cost }}=2.3,5.2$ and $10.7 \mathrm{mV}$ respectively. . . . . . . . . . . . . . .

9 Three different resistivity models are used to approximate the one shown in Fig. 7. The models are increasingly smoothed in order to introduce clutter to the data. It is evident that first level approximations of the resistivity structure are adequate for recovering the coordinates of the dipole. . . . . . . . . . . . . . .

10 Three different measurement steps are used for the numerical experiment showing in Fig. 7. Gaussian noise with standard deviation $2.5 \mathrm{mV}$ is added to the data. It is evident that the proposed method is robust for reasonable

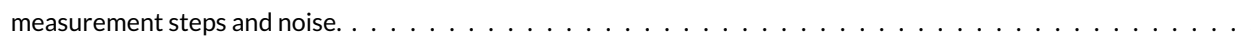

11 The observed (circles) and the calculated (solid line) SP anomalies using data from a geophysical investigation that took part in Chalkidiki, northern Greece (Vargemezis et. al , 2012). Down: The resulting potential distribution due to the normal fault. Up: The resulting potential distribution with both faults. The faults are depicted as black lines. The position of the borehole is highlighted with a blue star. . . . . . . . . . . . . . . . . . . . 
12 Blue circle illustrates the measurement point. Red and green triangles correspond to the two edges of the sheetlike body while $\rho_{1}$ and $\rho_{2}$ are the resistivity values of the first and second layer respectively. .

13 Comparison between analytical and numerical solutions for two-layered media using the grid-independent FD method (Spitzer et. al , 1999).

14 Numerical (circles) and analytical (solid lines) measurements over a normal fault located at a resistive background with $\rho_{2}=300$ ohm.m. The resistivity of the overburden layer varies from $\rho_{1}=0.05-2 \mathrm{ohm} . \mathrm{m}$. The width of the first layer is 20 meters. 


\section{REFERENCES}

Abramowitz, M. and Stegun, I., 1964. Handbook of mathematical functions with formulas, graphs, and mathematical tables, Applied mathematics series (National Bureau of Standards), Washington, D.C.: U.S. G.P.O.

Akgun, M., 2001. Estimation of some bodies parameters from the self-potential method using Hilbert transform, Journal of the Balkan Geophysical society, 4, 24-44.

Aizawa, K., 2004. A large self-potential anomaly and its changes on the quiet Mt. Fuji, Japan, Geophysical Research Letters, 31, 1-4.

Arfkens, G. B., \& Weber, H. J., 2001, Mathematical methods for physicists, 5th edition, Acedemic press.

Atchuta, R.D., Rambabu, H.V. and Sivakumar S.G.D.R., 1982. Fourier Transform Method for the interpretation of self-potential anomalies due to two dimensional inclined sheets of finite depth extent, Pure and Applied Geophysics, 120, 365-376.

Başokur, A. T., Akça, I. and Siyam, N. W. A. 2007. Hybrid genetic algorithms in view of the evolution theories with application for the electrical sounding method, Geophysical Prospecting, 55, 393-406.

Başokur, A. T. and Akca, í., 2011. Object-based model verification by a genetic algorithm approach: application to archaeological targets, Journal of Applied Geophysics, 74, 167-174.

Bhattacharya, B. B. and Roy, N., 1981. A note on the use of a nomogram for self-potential anomalies, Geophysical Prospecting, 29, 102-107.

Birch, F. S., 1998. Imaging the water table by filtering self-potential profiles, Ground Water, 36, (5), 779-782.

Biswas, A. and Sharma, S. P., 2014. Optimization of self-potential interpretation of 2-D inclined sheet-type structures based on very fast simulated annealing and analysis of ambiguity, Journal of Applied Geophysics, 105, 235-247.

Biswas, A. and Sharma, S. P., 2014. Resolution of multiple sheet-like structures in self-potential measurement, J. Earth Syst. Sci., $123,809-825$.

Biswas, A., 2015. Interpretation of residual gravity anomaly caused by simple shaped bodies using very fast simulated annealing global optimization, Geoscience Frontiers, 6, 875-893.

Constable, S. C., Parker, R. C. and Constable, G. G., 1987. Occam's inversion: A practical algorithm for generating smooth models from EM sounding data, Geophysics, 52, 289-300.

Corwin, R. F. and Hoover, D. B., 1979. The self-potential method in geothermal exploration, Geophysics, 44, $226-245$.

Dey, A. and Morrison, H.F., 1979. Resistivity modeling for arbitrarily shaped two-dimensional structures, Geophysical Prospecting, 27, 106-136.

Dieterich, J. M., Hartke, B., 2000. Empirical Review of Standard Benchmark Functions Using Evolutionary Global Optimization, Applied Mathematics, 3, 1552-1564.

Dwa, S. and Warnana D. D., 2018. Black hole algorithm for determining model parameter in self-potential data, Journal of Applied Geophysics, 148, 189-200.

Eppelbaum, V. L. and Khesin, B., 2012. Geophysical studies in the Caucasus, Springer.

Essa, S. K. and Elhussein, M., 2017. A new approach for the interpretation of self-potential data by 2-D inclided plate, Journal of Applied Geophysics, 136, 455-461.

Fournier, C., 1989. Spontaneous potentials and resistivity surveys applied to hydrogeology in a volcanic area: case history of the Chaine des Puys (Puy-de-dome, France), Geophysical Prospecting, 37, 647-668. 
Giannakis, I., Tsourlos, P., Papazachos, C. and Vargemezis, G., 2012. Modeling and inversion of self-potential anomalies due to sheet-like bodies under the presence of arbitary 2-D resistivity distributions, in Proc. 18th European Meeting of Environmental and Engineering Geophysics, Paris, France.

Grech, R., T. Cassar, J. Muscat, K. P. Camilleri, S. G. Fabri, M. Zervakis, P. Xanthopoulos, V. Sakkalis, and B. Vanrumste, 2008. Review on solving the inverse problem in EEG source analysis, Journal of NeuroEngineering and Rehabilitation, 5, no. 25.

Gokturkler, G. and Balkaya, C. 2012. Inversion of self-potential anomalies caused by simple-geometry bodies using global optimization algorithms, J. Geophys. Eng., 9, 498-507.

Haas, A. K., Revil, A., Karaoulis, M., Frash, L., Hampton, J., Gutierrez, M., and Mooney, M, 2013. Electrical potential source localization reveals a borehole leak during hydraulic fracturing, Geophysics, 78, 2, D93-D113.

Haijun, Y., Yongzhong, X., Gengxin, P., Guiping, Y., Meng, C. Wensheng, D., Yongfeng, Z., Yongfu, C. and Xingjun, W., 2017. Particle swarm optimization and its application to seismic inversion of igneous rocks, International Journal of Mining Science and Technology, 27, 349-357.

Hansen, P. C., 1992. Analysis of discrete ill-posed problems by means of the L-curve, SIAM Review, 34, 561-580.

Hansen, P. C., 1993. The use of the L-curve in the regularization of discrete ill-posed problems, SIAM J. Sci. Comput., 14, 14871503.

Heinshon, G., White, A., Robinson, D. and Fathianpour, N. 2005. Marine self-potential gradient exploration of the continental margin, Geophysics, 70, (5), G109-G118.

Hesham, M., Kaliouby, E., Mansour, A. and Garni, A., 2009. Inversion of self-potential anomalies caused by 2D inclined sheets using neural networks, Journal of Geophysics and Engineering 6, 29-34.

Jagannadha Rao, S., Rama Rao, P. and Radhakrishna Murthy, I. V., 1993. Automatic inversion of self-potential anomalies of sheet-like bodies, Computers \& Geosciences, 19, 61-73.

Jouniaux, L., Maineult, A., Naudet, V., Pessel, M. and Sailhac, P., 2009. Review of self-potential methods in hydrogeophysics, Applied Geophysics, 928-936.

Kawada, Y. and Kasaya, T., 2017. Marine self-potential survey for exploring seafloor hydrothermal ore deposits, Nature, 7, 112.

Karaoulis, M., Revil, A., Minsley, B., Todesco, M., Zhang, J. and Werkema D. D., 2014. Time-lapse gravity inversion with an active time constraint, Geophysical Journal International, 196, (2), 748-759.

Kelley, D. F., Destan, T. J. and Luebbers, R. J., 2007. Debye function expansions of complex permittivity using a hybrid particle swarm-least squares optimisation approach, IEEE Trans. Antennas Propag. , 55, (7), 1999-2005.

Kennedy, J. and Eberhart, R. C., 1995. Particle swarm optimization in Proc. IEEE Int. Conf. Neural Netw., 4, $1942-1948$.

Kennedy, J. and Spears, W. M., 1998. Matching algorithms to problems: an experimental test of the particle swarm and some genetic algorithms on multi modal problem generator, in Proc. IEEE Int. Conf. Evolutionary Computation.

Last, B. J and Kubik, K., 1983. Compact gravity inversion, Geophysics, 48, 713-721.

Li, Y. and Oldenburg, D. W., 1996. 3-D inversion of magnetic data, Geophysics, 61, (2), 394-408.

Li, Y. and Oldenburg, D. W., 1998. 3-D inversion of gravity data, Geophysics, 63, (1), 109-119.

Maio, R. D., Rani, P., Piegari, E. and Milano, L., 2016. Self-potential data inversion through a genetic-price algorithm, Computers $\&$ Geosciences, 94, 86-95. 
Mao, D., Revil, A., Hort, R. D., Munakata-Marr, J., Atekwana, E. A. and Kulessa, B., 2015. Resistivity and self-potential tomography applied to groundwater remediation and contaminant plumes: Sandbox and field experiments, Journal of Hydrology, 530, 1-14.

Marquadt, D.W., 1963. An algorithm for least-squares estimation of nonlinear parameters, Journal of the society for industrial and applied mathematics, 11,431-441.

Marshall, D.J., and Madden, T. R., 1959. Induced polarization, a study of its causes, Geophysics, 24, (4), 790-816.

Martinez, J. L. F., Gonzalo, E. G., Alvarez, J. P. F., Kuzma, H. A. and Perez, C. O., 2010. PSO: A powerful algorithm to solve geophysical inverse problems Application to a 1D-DC resistivity case, Journal of Applied Geophysics, 71, 13-25.

Meiser, P., 1962. A method of quantitative interpretation of self-potential measurements, Geophysical Prospecting, 10, 203218.

Minsley, B. J., 2007. Modeling and inversion of self-potential data, PhD thesis submitted to the Massachusetts Institute of Technology.

Minsley, B. J., Sogade, J. and Morgan F. D., 2007. Three-dimensional source inversion of self-potential data, Journal of Geophysical Research, 112, 1-13.

Minsley, B. J., Coles, D. A., Vichabian, Y. and Morgan, F. D., 2008. Minimization of self-potential survey mis-ties acquired with multiple reference locations, Geophysics, 73, (2), F71-F81.

Naudet, V., Revil, A. and Bottero, J. Y., 2003. Relationship between self-potential (SP) signals and redox conditions in contaminated groundwater, Geophysical Research Letters, 30, (21), 1-4.

Nostrand, R. G. and Cook, K. L., 1966. Interpretation of Resistivity Data, United States Government Printing Office.

Nyquist, J. E. and Corry, C. E., 2002. Self-potential: the ugly duckling of environmental geophysics, The Leading Edge, 446-451.

Pallero, J. L. G., Martinez, J. L. F., Bonvalot, S. and Fudym, O., 2015. Gravity inversion and uncertainty assessment of basement relief via Particle Swarm Optimization, Journal of Applied Geophysics, 116, 180-191.

Pallero, J. L. G., Martinez, J. L. F., Bonvalot, S. and Fudym, O., 2017. 3D gravity inversion and uncertainty assessment of basement relief via Particle Swarm Optimization, Journal of Applied Geophysics, 139, 338-350.

Pedersen, L. B., 1977. Interpretation of potential field data a generalized inverse approach, Geophysical Prospecting, 25, 199230.

Peksen, E., Yas, T., Kayman, A. Y. and Özkan, C. 2011. Application of particle swarm optimization on self-potential data, Journal of Applied Geophysics, 75, 305-318.

Portniaguine, O. and Zhdanov, S. M., 1999. Focusing geophysical inversion images, Geophysics, 64, 874-887.

Rao, A. D. and Babu, R. H. V., 1983. Quantitative interpretation of self-potential anomalies due to two-dimensional sheet-like bodies, Geophysics, 48 1659-1664.

Robinson, J. and Rahmat-Samii, Y., 2004. Particle swarm optimization in electromagnetics, IEEE Trans. Antennas Propag., 52, 397--407.

Santos, F. A. M. 2010. Inversion of self-potential of idealized bodies anomalies using particle swarm optimization, Computers \& Geosciences 36, 1185-1190.

Sato, M. and Mooney, H. M., 1960, The electrochemical mechanism of sulfide self-potentials, Geophysics, 25, (1), $226-249$.

Sharma, P. V., 1997. Enviromental and Engineering Geophysics, Cambridge University Press. 
Shao, Z., Wang, D., Wang, Y. and Zhong, X., 2014. Theory and application of magnetic and self-potential methods in the detection of the Heshituoluogai coal fire, China, Journal of Applied Geophysics, 104, 64-74.

Shao, Z., Wang, D., Wang, Y., Zhong, X., Zhang, Y. and Song, W., 2017. Experimental study of the self-potential anomaly caused by coal fires, Journal of Applied Geophysics, 145, 124-132.

Shaw, R. and Srivastava, S., 2007. Particle swarm optimization: A new tool to invert geophysical data Geophysics, 72, (2), F75F83.

Shi, Y. and Eberhart, R., 1998, A modified particle swarm optimizer, IEEE International Conference on Evolutionary Computation Proceedings. IEEE World Congress on Computational Intelligence (Cat. No.98TH8360), Anchorage, AK, USA, 69-73.

Soupios, P., Akca, I., Mpogiatzis, P., Basokur, A., T and Papazachos, C., 2011. Application of hybrid genetic algorithms in seismic tomography Journal of Applied Geophysics, 75, 479-489.

Spitzer, K., Chouteau, M. and Boulanger, O., 1999. Grid-independent electrode positioning for 3D DC resistivity and IP forward modelling, Extended Abstracts Book, 2nd Int. Symp. on Three-Dimensional Electromagnetics, University of Utah, Salt Lake City, UT, 189-192.

Tang, J., Wang, F., Xiao, X. and Zhang, L., 2011. 2.5-D DC Resistivity Modelling Considering Flexibility and Accuracy, Journal of earth science, 22, 124-130.

Tikhonov, A. and Arsenin, V. Y., 1977. Solution of ill-posed problems, V. H. Winston and Sons.

Turcotte, D. L., 1992. Fractals and chaos in geology and geophysics, The Press Syndicate of the University of Cambridge.

Vargemezis, G., Tsourlos, P., Stampolidis, A., Fikos, I., Ballas, D. and Papadopoulos N., 2012. A focusing approach to ground water detection by means of electrical and EM methods: the case of Paliouri, Northern Greece, Stud. Geophys. Geod., 56, i-xvi.

Xu, S. Z, Duan, B. and Zhang, D., 2000. Selection of the wavenumbers k using an optimization method for the inverse Fourier transform in 2.5D electrical modeling, Geophysical Prospecting, 48, 789-796. 


\section{LIST OF FIGURES}



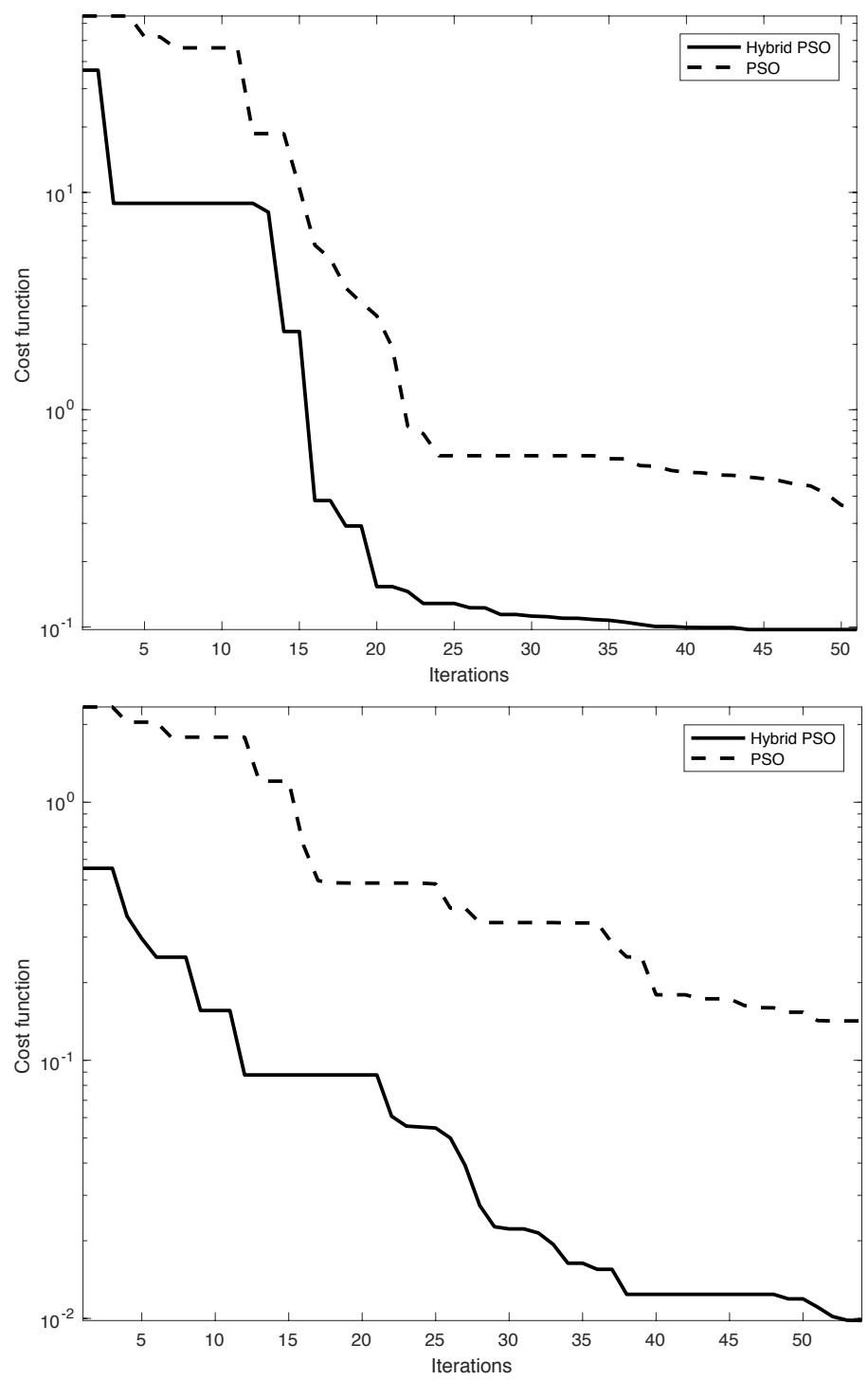

FIGURE 1 Convergence rate of the hybrid PSO compared to simple PSO. Up: The model is a two layered medium with $\rho_{1}=50 \mathrm{ohm} . \mathrm{m}, \rho_{2}=100 \mathrm{ohm} . \mathrm{m}$ and $h=10 \mathrm{~m}$. A tilted sheet-like body is placed in the first layer with current intensity $I=-3 \mathrm{~mA}$. Down: The model is a two layered media with $\rho_{1}=300$ ohm.m, $\rho_{2}=100$ ohm.m and $h=20 \mathrm{~m}$. Two normal to the surface sheet-like bodies are placed parallel to each other with $10 \mathrm{~m}$ distance. In both examples the parameters of PSO are, $w=1, c 1=1, c 2=0.5$ and the number of particles equals to 40 . 

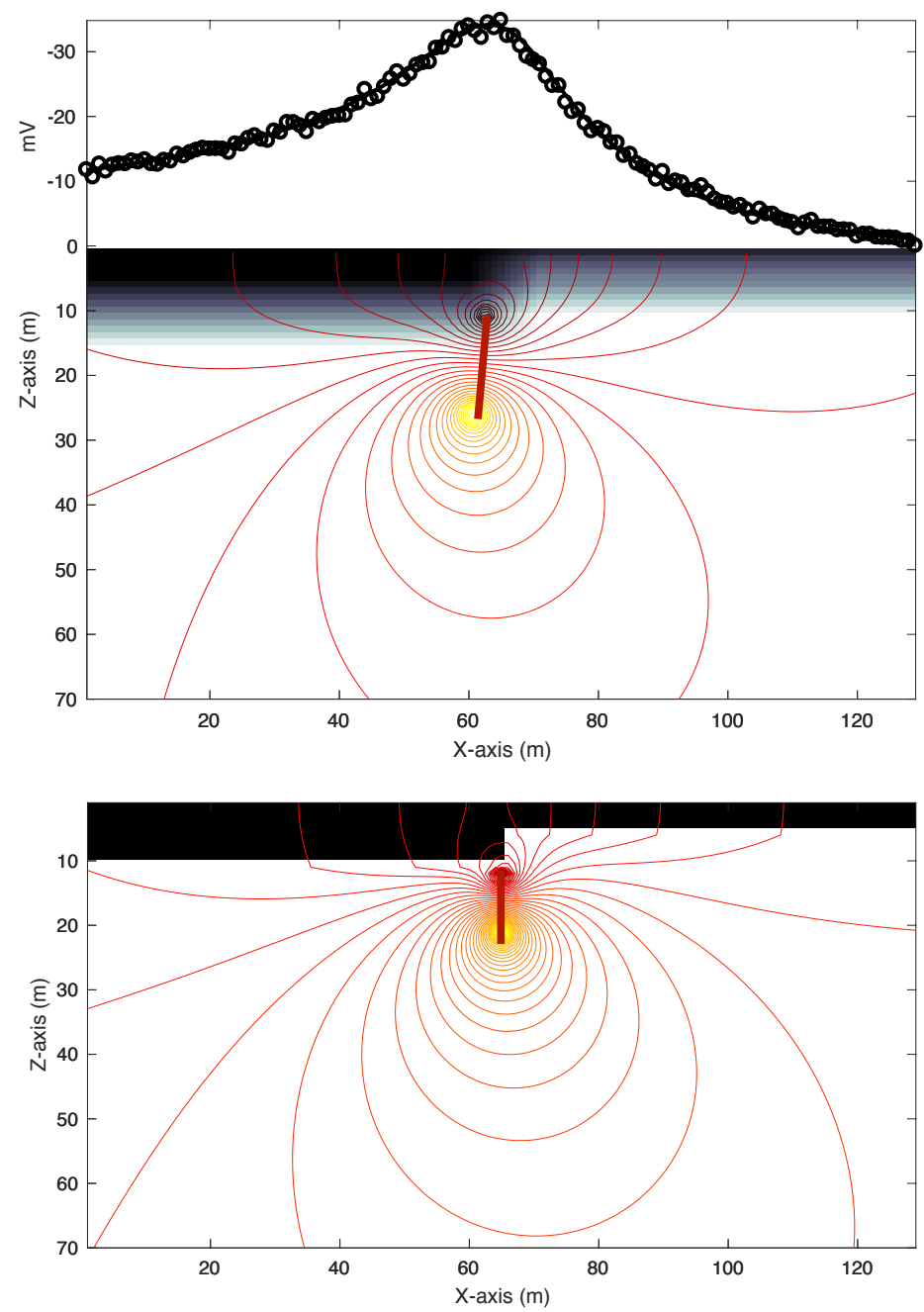

FIGURE 2 Down: The self-potential anomaly generated by a normal fault. The resistivity of the bedrock and the overburden layer is $500 \mathrm{ohm} . \mathrm{m}$ and $40 \mathrm{ohm} . \mathrm{m}$ respectively. Up: The resulting sheet-like body using the proposed scheme subject to a smoothed resistivity distribution. The observed data are illustrated with circles while the calculated SP anomaly with solid line. 


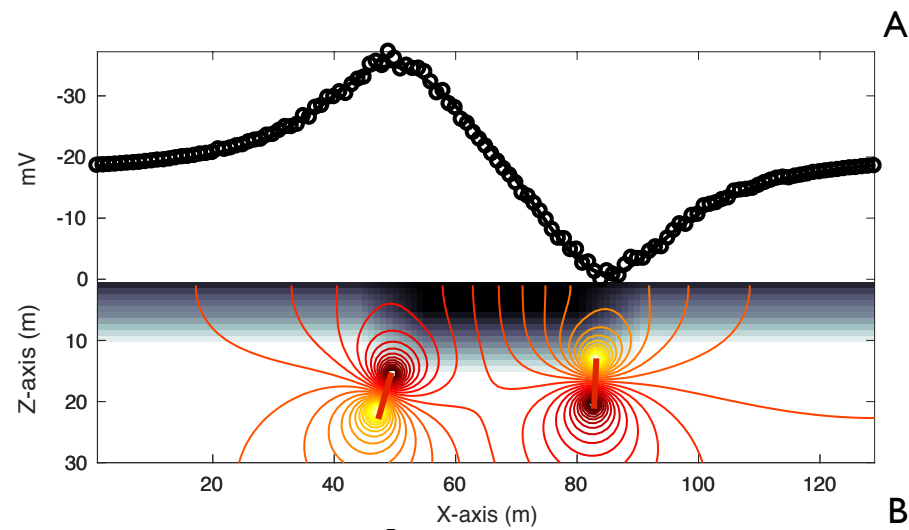

A.
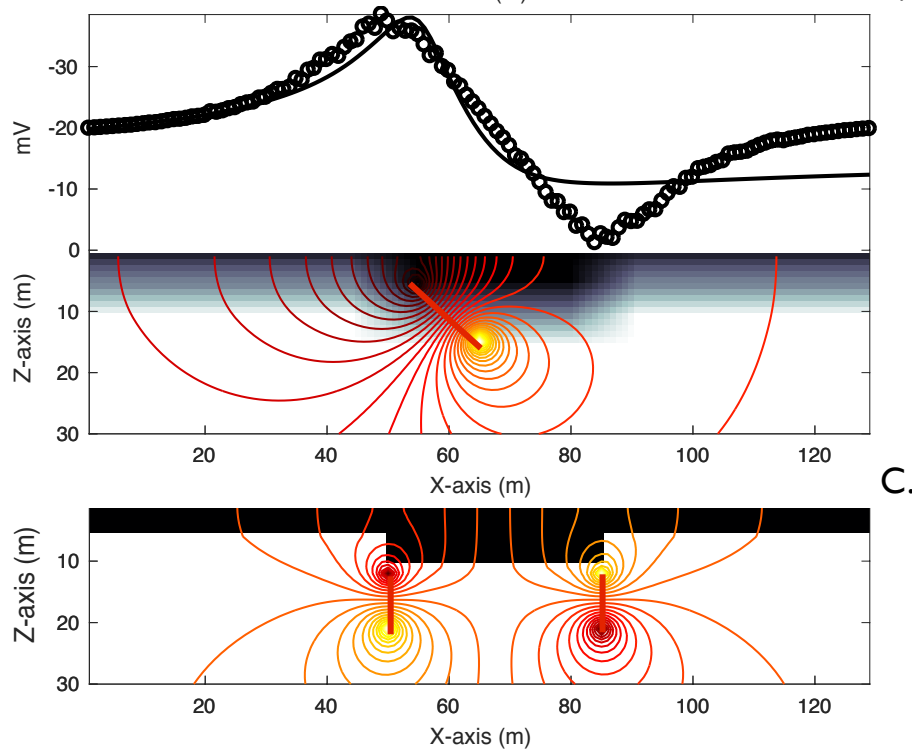

FIGURE 3 C: The self-potential anomaly generated by two normal faults. The resistivity of the bedrock and the overburden layer is $200 \mathrm{ohm} . \mathrm{m}$ and $70 \mathrm{ohm} . \mathrm{m}$ respectively. B: The resulting sheet-like body using the proposed scheme subject to a smoothed resistivity distribution. The observed data are illustrated with circles while the calculated SP anomaly with solid line. A: Similar to B using two sheet-like bodies. 

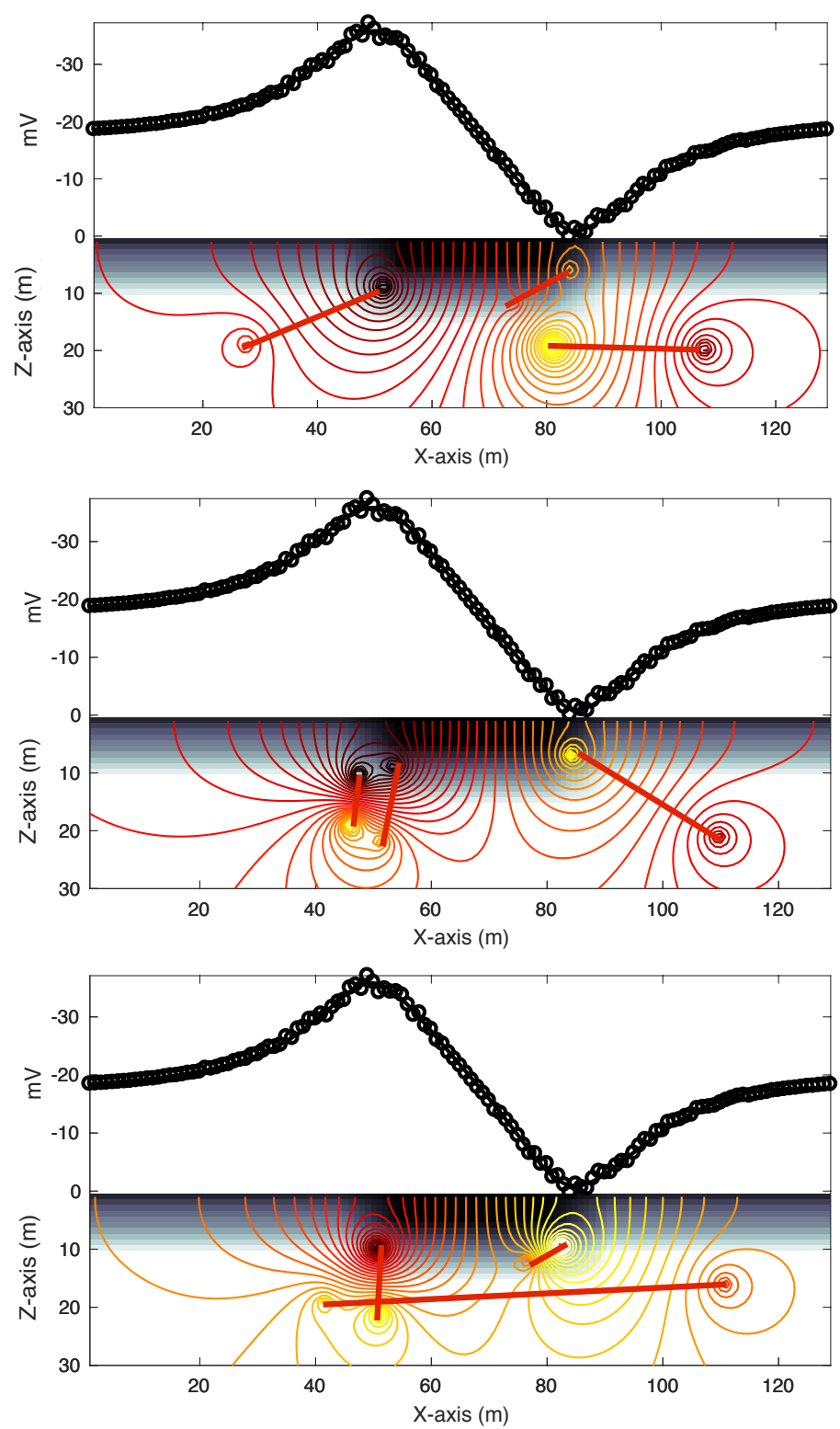

FIGURE 4 The observed and the calculated SP anomaly are illustrated with circles and solid lines respectively. The model is similar to the one described in Figure 3. Three different cases are shown that sufficiently fit the measured data. This highlights the fact that inverting for multiple sheet-like bodies requires adequate information (data or constrains) in order to tackle the non-uniqueness embedded in SP measurements. 

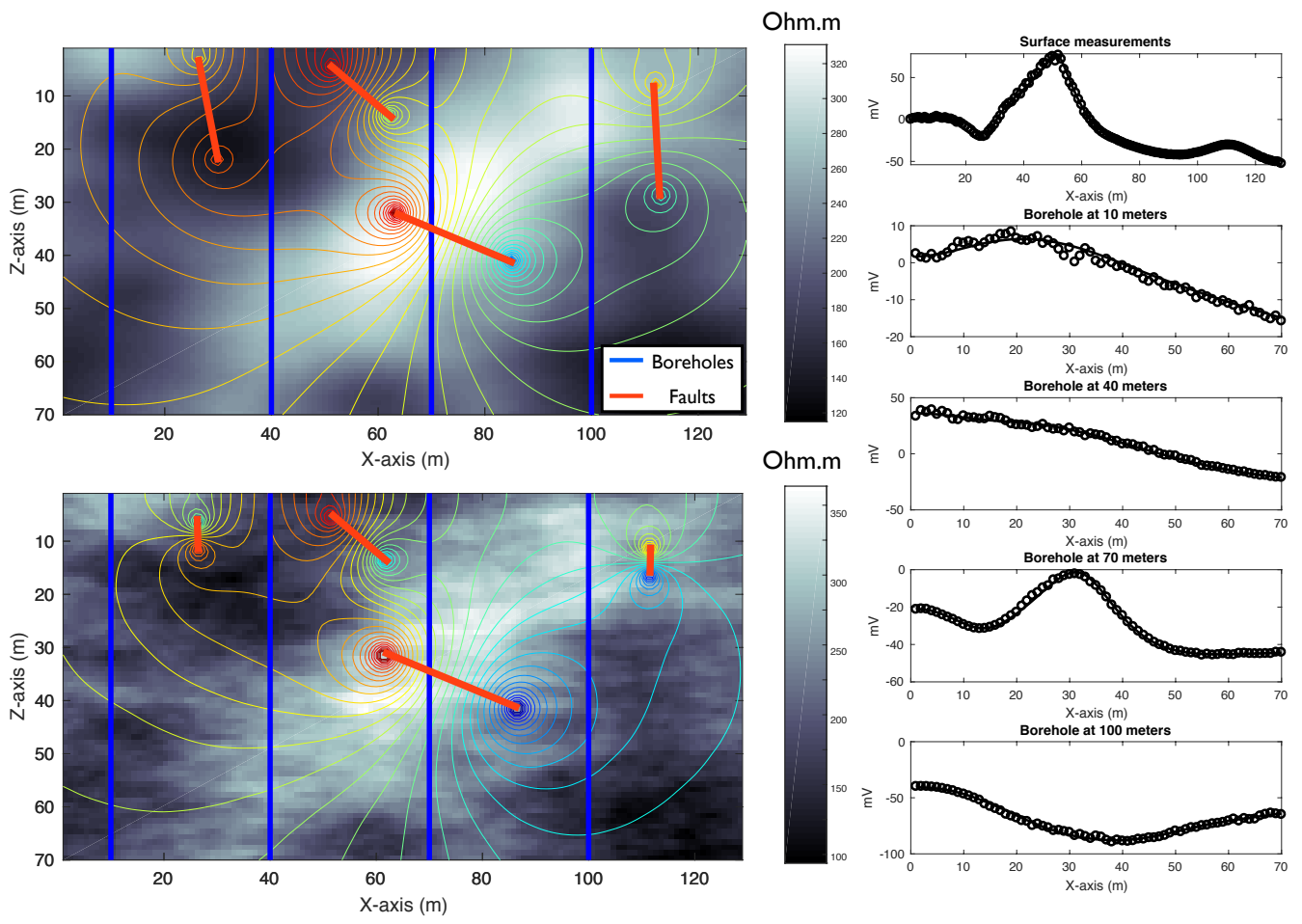

FIGURE 5 Left Down: The self-potential anomaly generated by four dipoles. A fractal correlated noise is used to generate a realistic resistivity distribution. Left Up: The resulting sheet-like bodies using the proposed scheme subject to a smoothed resistivity distribution. On the right, the observed and calculated data are illustrated with circles and solid lines respectively. 

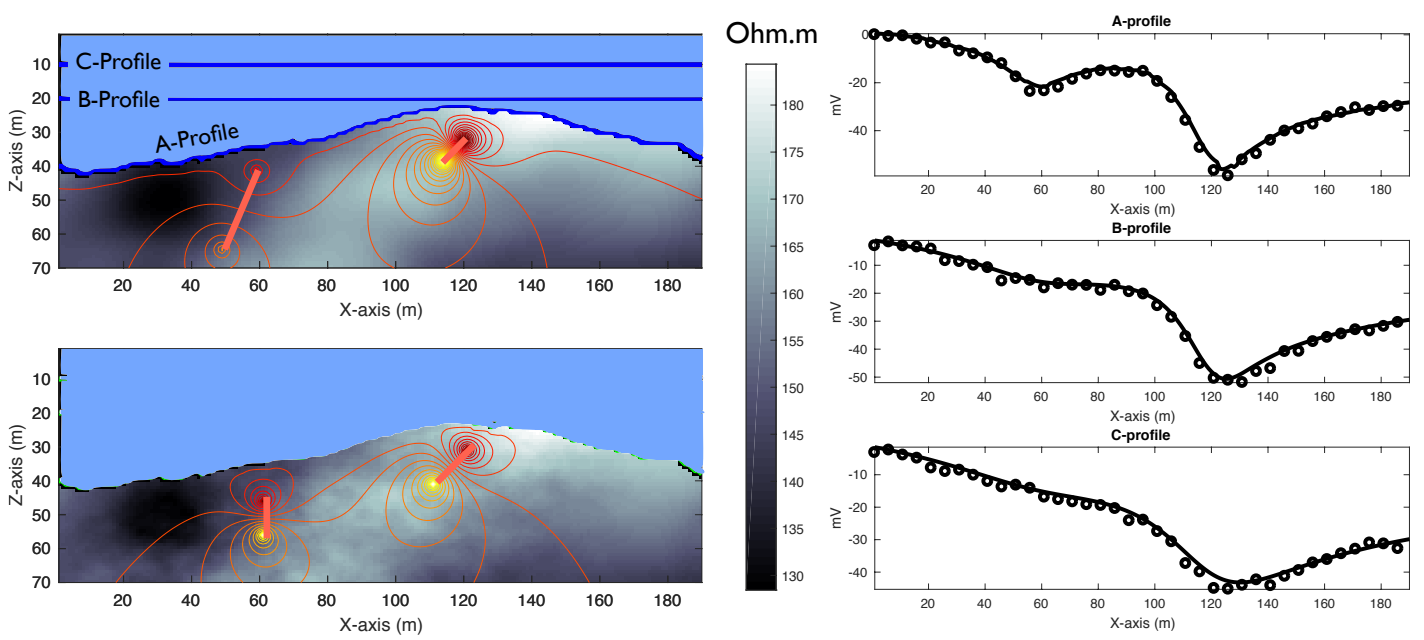

FIGURE 6 Left Down: The self-potential anomaly generated by two dipoles in a marine environment. The water mass is illustrated with light blue colour. The resistivity of the water is $1 \mathrm{ohm}$.m. Left Up: The resulting sheet-like bodies using the proposed scheme subject to a smoothed resistivity distribution. On the right, the observed and calculated data are illustrated with circles and solid lines respectively. 


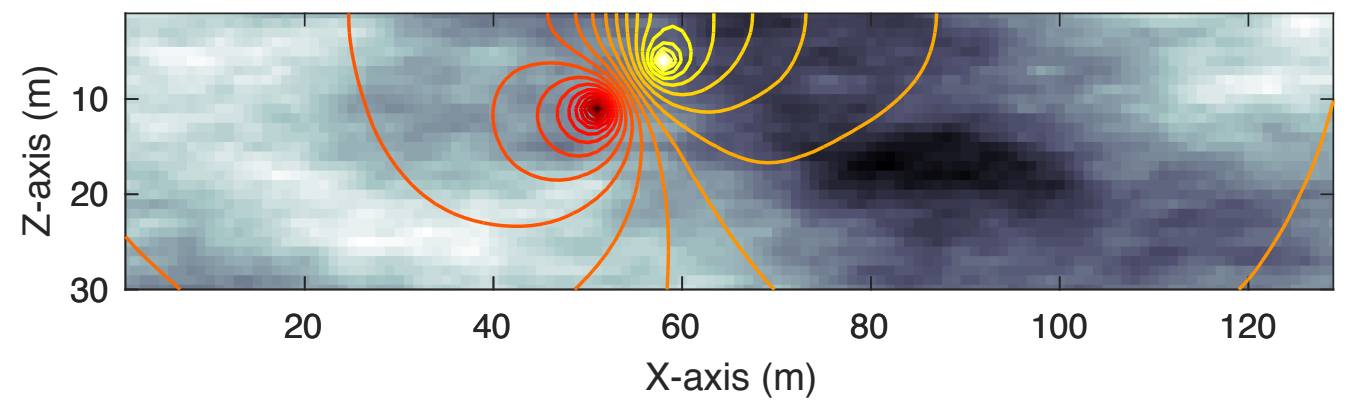

Ohm.m

100

FIGURE 7 The actual model used to evaluate the sensitivity of the current technique to noise and clutter. A single tilted dipole is placed inside a fractal resistivity distribution which varies from 30-330 ohm.m. The measurement-step equals to 1 meter. 


\section{Ohm.m}
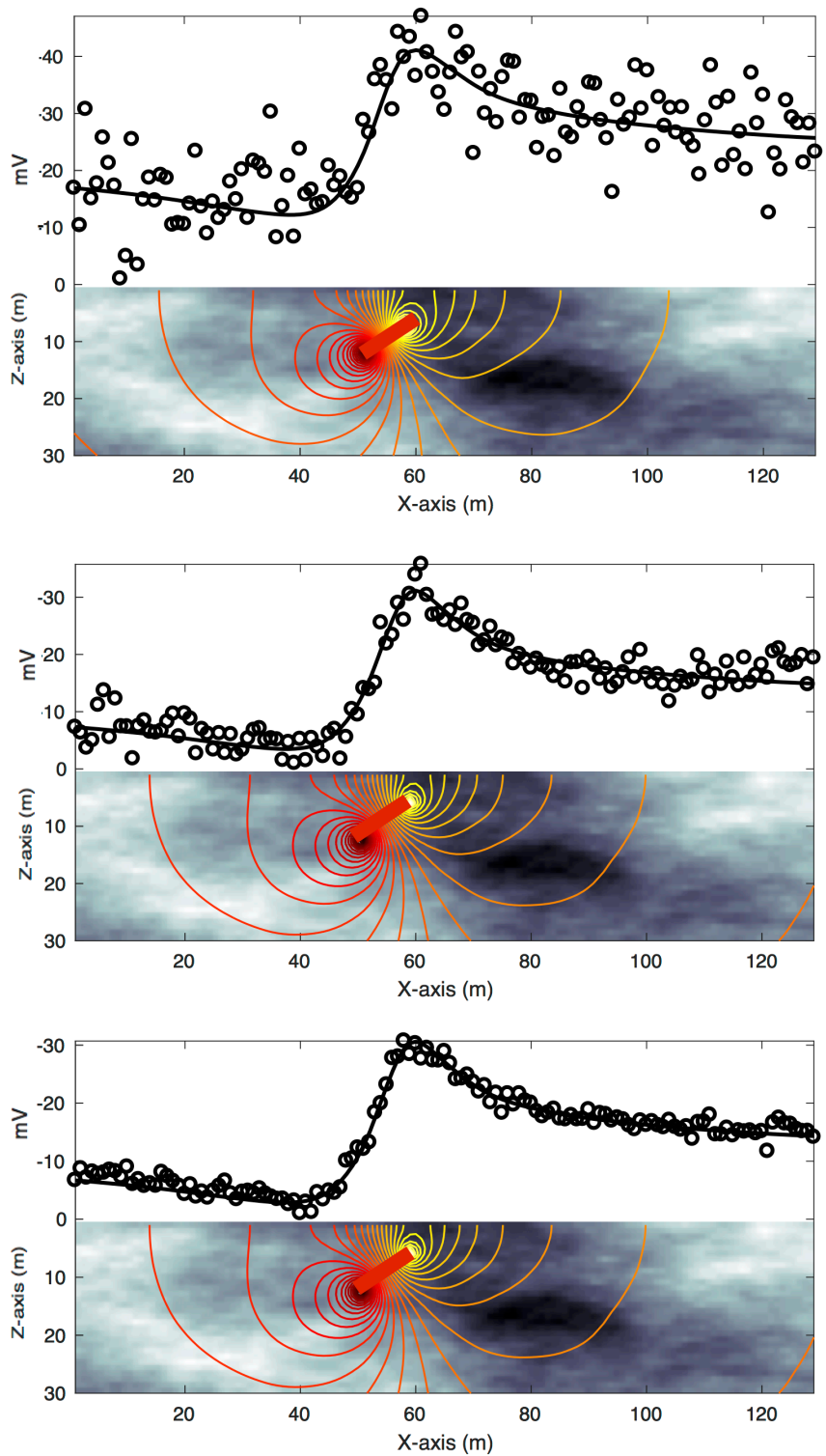

FIGURE 8 The hybrid scheme is applied to data with different levels of noise. The inverted dipole is illustrated with red line. The actual position of the dipole is shown in Fig. 7. The data are corrupted with Gaussian noise with 2.5,5 and $10 \mathrm{mV}$ standard deviation. It is evident that the proposed scheme can affectively deal with Gaussian noise (in the presence of dense measurements) and accurately recover the coordinates of the sheet-like body. Due to the sufficient number of measurements, the squared root of the misfit function (16) is close to the standard deviation of the noise, in particular $\sqrt{\text { Cost }}=2.3,5.2$ and $10.7 \mathrm{mV}$ respectively. 


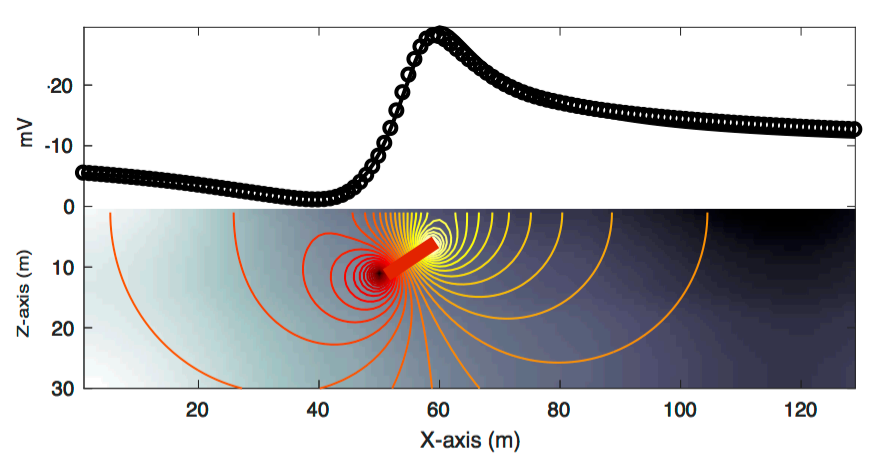

Ohm.m

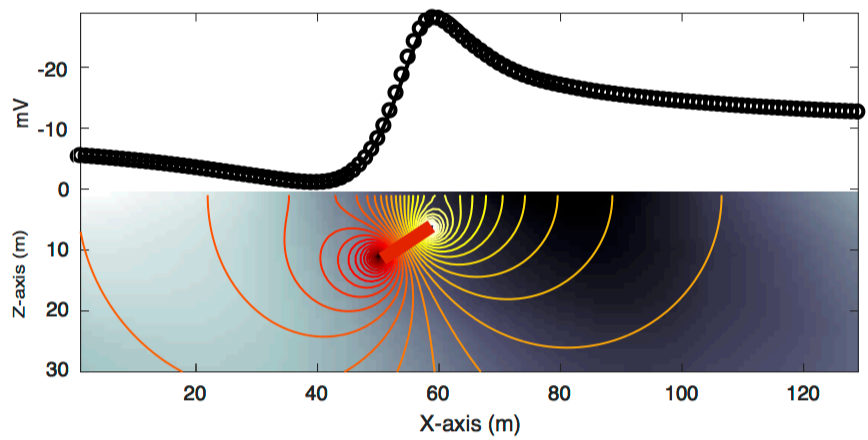

300

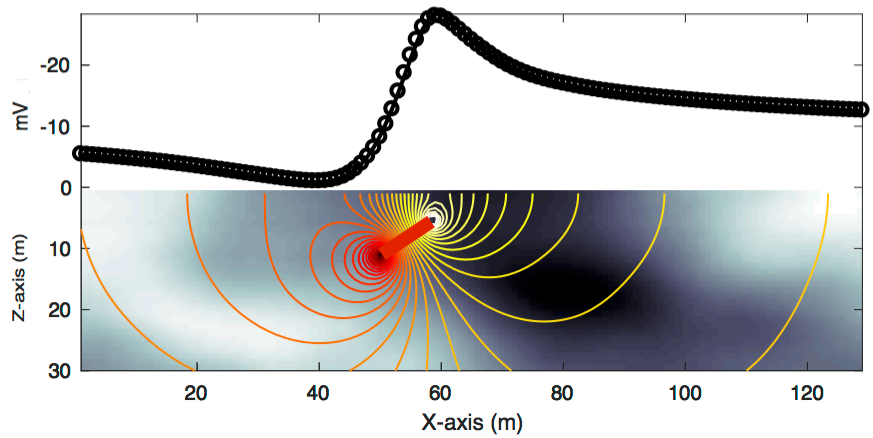

FIGURE 9 Three different resistivity models are used to approximate the one shown in Fig. 7. The models are increasingly smoothed in order to introduce clutter to the data. It is evident that first level approximations of the resistivity structure are adequate for recovering the coordinates of the dipole. 


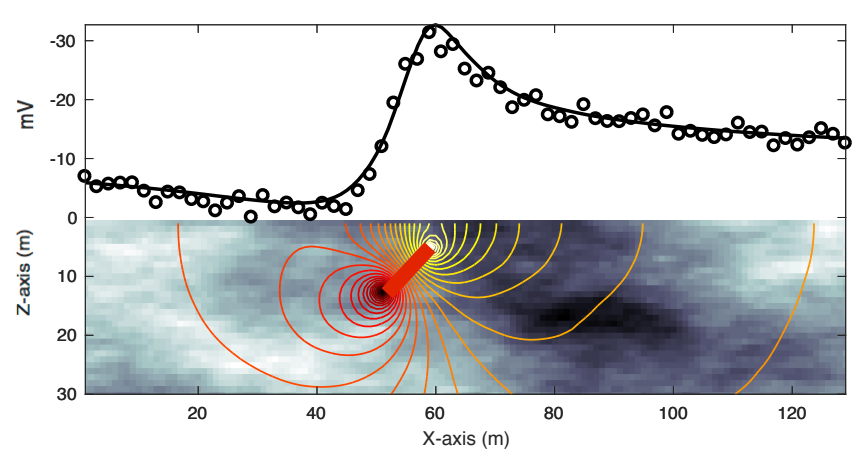

\section{Ohm.m}
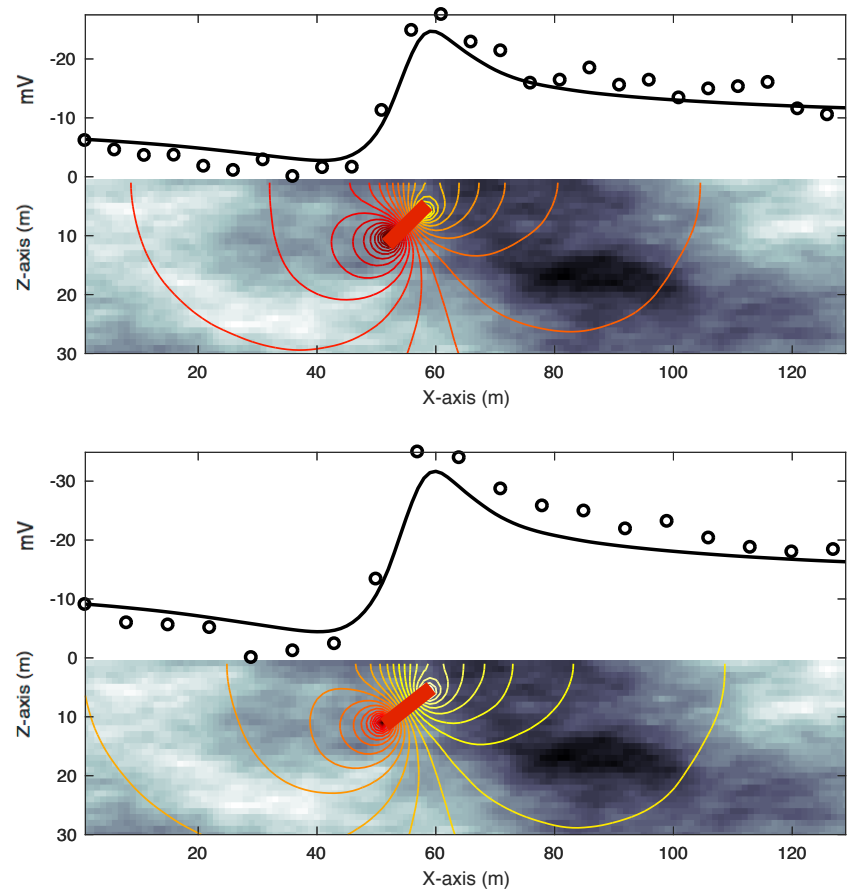

FIGURE 10 Three different measurement steps are used for the numerical experiment showing in Fig. 7. Gaussian noise with standard deviation $2.5 \mathrm{mV}$ is added to the data. It is evident that the proposed method is robust for reasonable measurement steps and noise. 


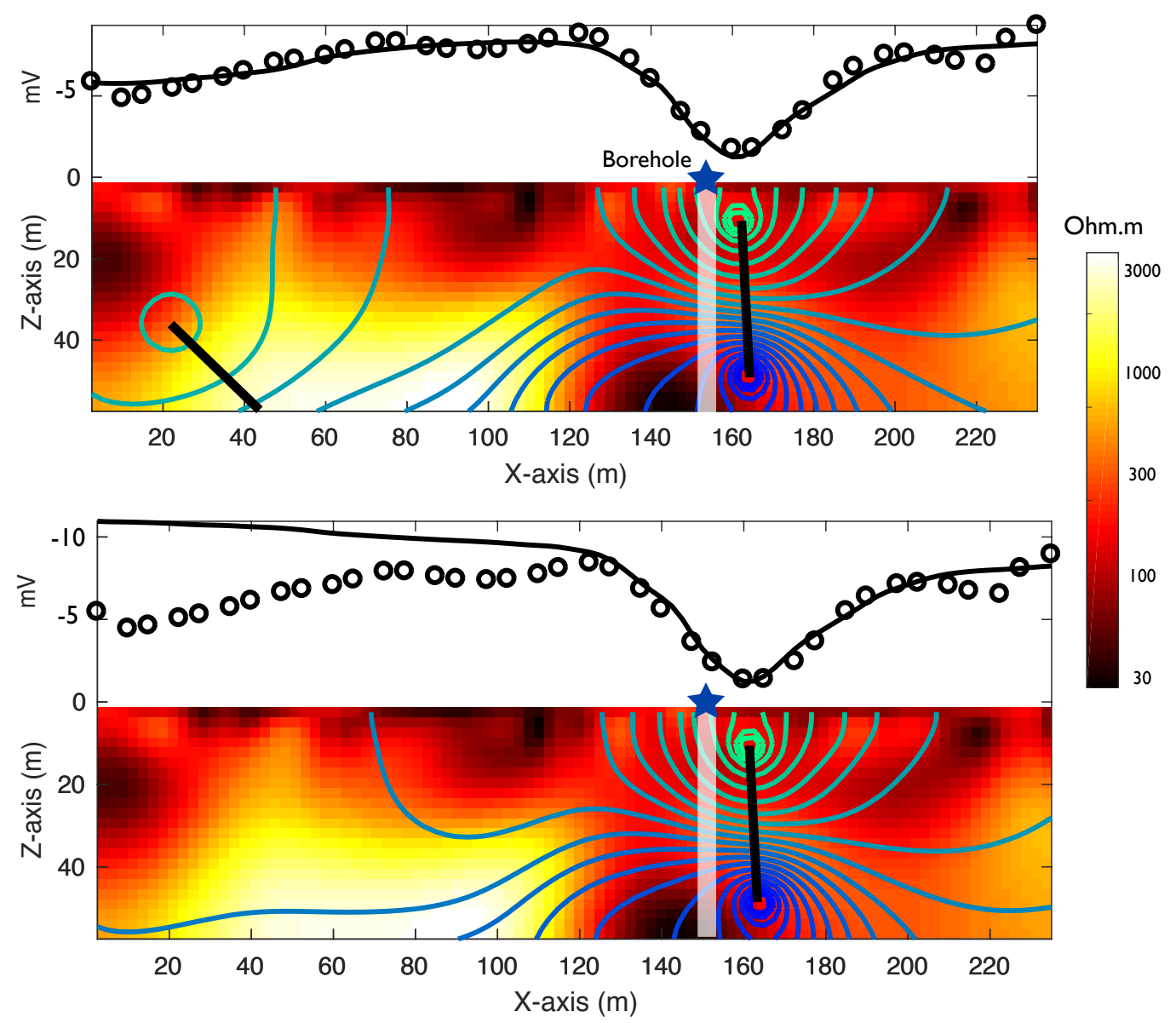

FIGURE 11 The observed (circles) and the calculated (solid line) SP anomalies using data from a geophysical investigation that took part in Chalkidiki, northern Greece (Vargemezis et. al , 2012). Down: The resulting potential distribution due to the normal fault. Up: The resulting potential distribution with both faults. The faults are depicted as black lines. The position of the borehole is highlighted with a blue star. 


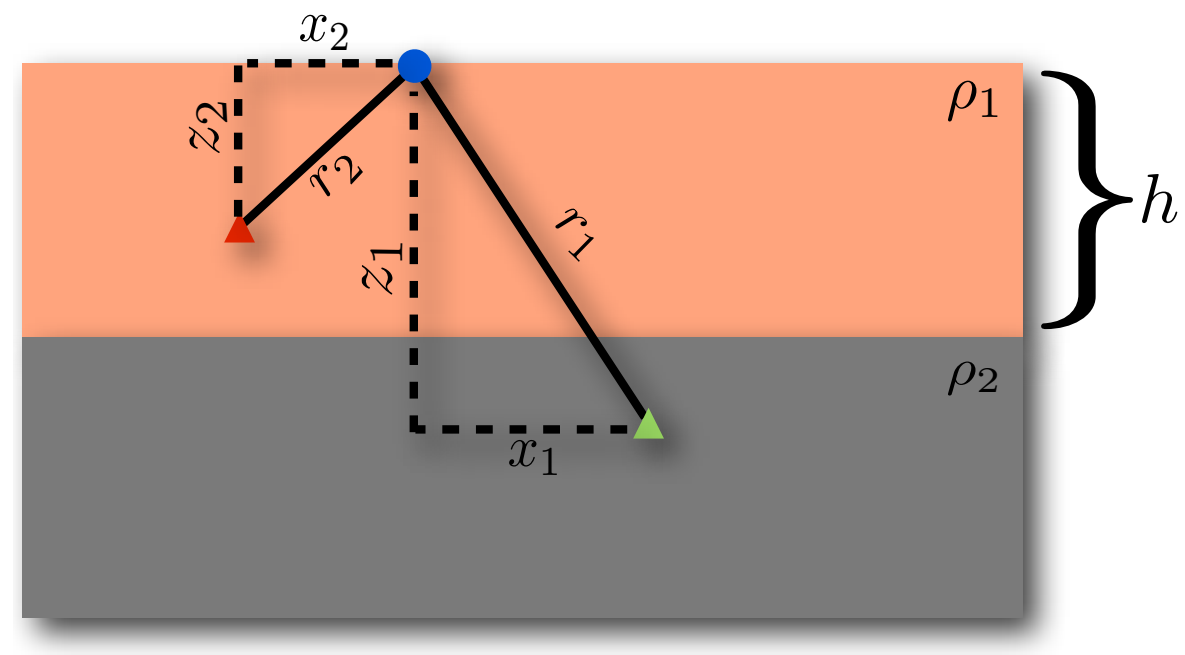

FIGURE 12 Blue circle illustrates the measurement point. Red and green triangles correspond to the two edges of the sheet-like body while $\rho_{1}$ and $\rho_{2}$ are the resistivity values of the first and second layer respectively. 

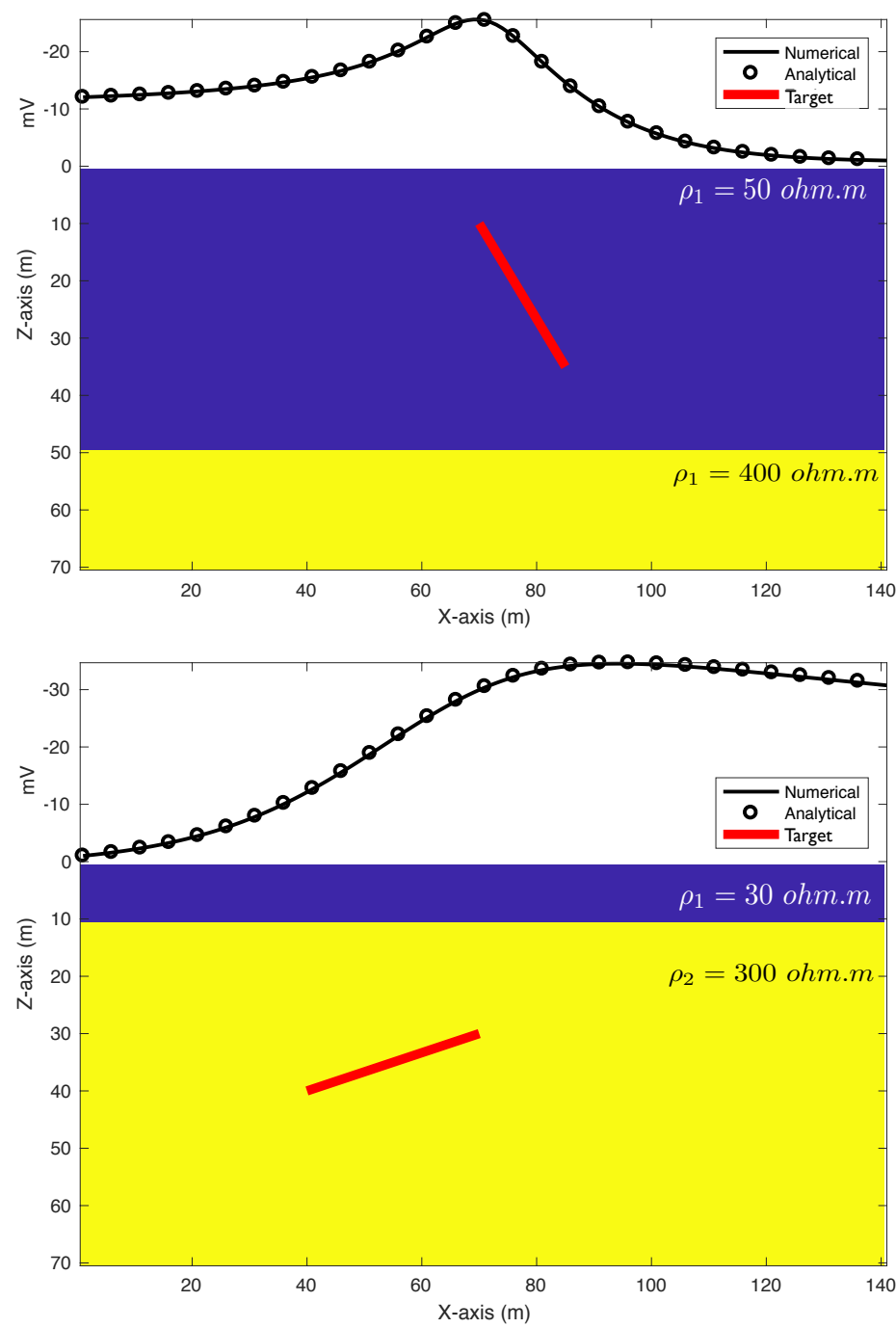

FIGURE 13 Comparison between analytical and numerical solutions for two-layered media using the grid-independent FD method (Spitzer et. al , 1999). 


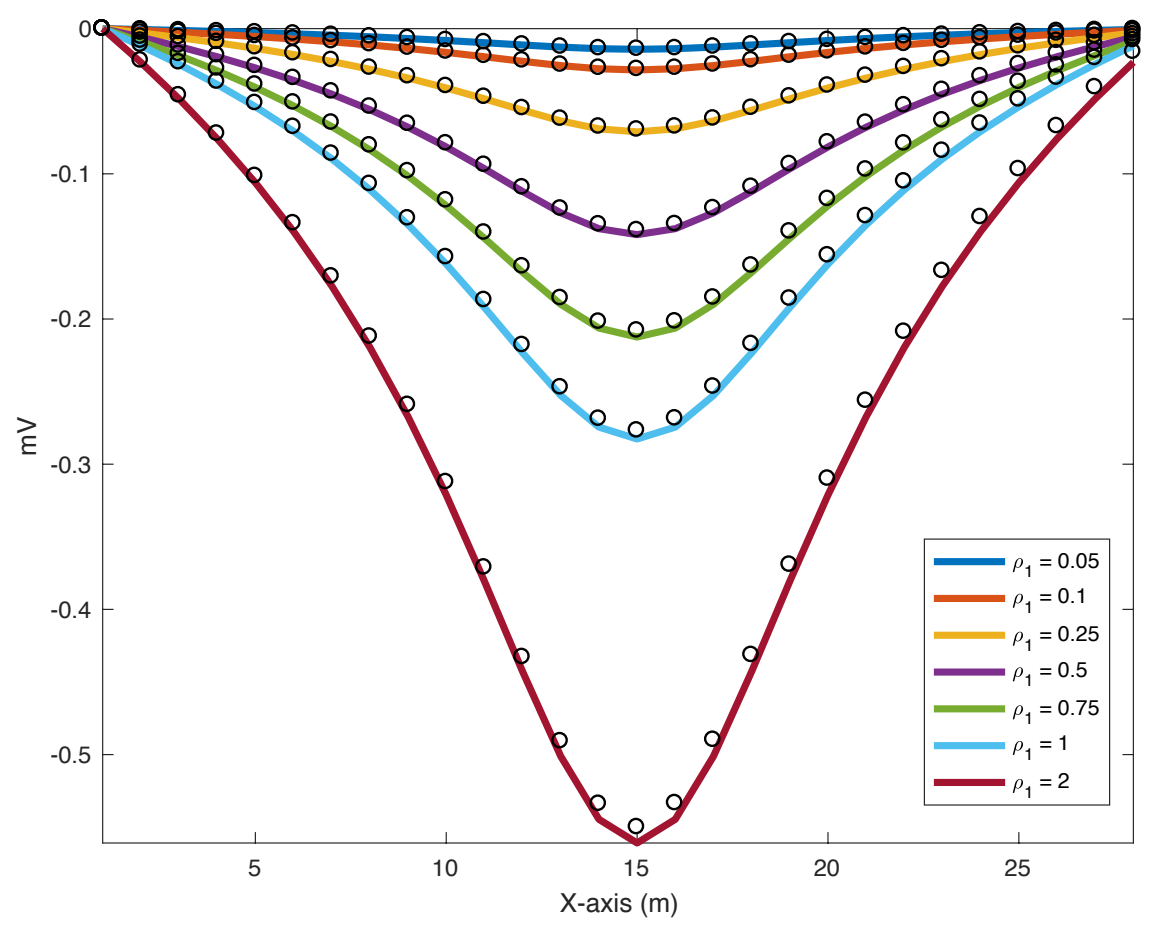

FIGURE 14 Numerical (circles) and analytical (solid lines) measurements over a normal fault located at a resistive background with $\rho_{2}=300 \mathrm{ohm}$.m. The resistivity of the overburden layer varies from $\rho_{1}=0.05-2 \mathrm{ohm} . \mathrm{m}$. The width of the first layer is 20 meters. 